BULLETIN Bulletin hispanique

HISPANIQUE Université Michel de Montaigne Bordeaux

110-1 | 2008

Varia

\title{
Poema del Cante Jondo
}

réévaluation d'une poétique en devenir

\section{Henry Gil}

\section{OpenEdition}

\section{Journals}

Édition électronique

URL : https://journals.openedition.org/bulletinhispanique/636

DOI : 10.4000/bulletinhispanique.636

ISSN : $1775-3821$

Éditeur

Presses universitaires de Bordeaux

\section{Édition imprimée}

Date de publication : 1 juin 2008

Pagination : 191-243

ISBN : 978-2-86781-511-9

ISSN : 0007-4640

Référence électronique

Henry Gil, « Poema del Cante Jondo », Bulletin hispanique [En ligne], 110-1 | 2008, mis en ligne le 01 juin 2011, consulté le 18 septembre 2021. URL : http://journals.openedition.org/bulletinhispanique/636 ; DOI : https://doi.org/10.4000/bulletinhispanique.636 


\title{
Poema del Cante Jondo : réévaluation d'une poétique en devenir
}

\author{
HenRY GIL \\ Université de Poitiers
}

À Marie-Claire Zimmermann

\begin{abstract}
Ce travail se propose d'analyser - en dehors de tout "folklorisme", mais sans renier pour autant les emprunts au chant primitif andalou - la nouveauté et la modernité de Poema del Cante Jondo, tout en mettant en évidence sa logique architecturale et sa spécificité poétique au sein de l'œuvre. Il s'agit donc de révéler les mécanismes et les significations d'une poétique de l'épure, de "l'élagage lyrique ", qui passe par l'élaboration d'une image poétique de type gongorin et par une relecture de la "copla" dans un sens épigrammatique afin d'inventer une métrique plus libre mais sans céder au vers-librisme. Une dernière partie consiste à cerner les diverses manifestations de la théâtralité et ses rapports avec le poétique au sein de ce recueil.
\end{abstract}

Este articulo se propone analizar - fuera de todo folklorismo, pero sin negar por ello los préstamos del canto primitivo andaluz - la novedad y modernidad de Poema del Cante Jondo, evidenciando su lógica arquitectónica y su especificidad poética dentro de la obra. Se trata pues de mostrar los mecanismos y significados de una poética de la depuración, de "la poda lírica", que pasa por la elaboración de una imagen poética de tipo gongorino y por la relectura de la copla en un sentido epigramático para inventar una métrica más libre pero sin ceder al versolibrismo. Una última parte consiste en delimitar las distintas manifestaciones de la teatralidad y sus relaciones con lo poético dentro de este poemario.

The aim of this paper is to analyse - in the process avoiding "folklorism " without denying borrowings from the original Andalusian song - the novelty and modernity of the Poema del Cante Jondo, while emphasizing its architectural structure and the

$B H i$, Tome $110, \mathrm{n}^{\circ} 1$ - juin 2008 - p. 191 à 243. 
specificity of its poetics within Lorca's production. The goal to reveal the mechanisms and meanings of a sharpened poetics, or "lyrical pruning", which involves the elaboration of a Gongorian style poetic image and the re-reading of the "copla" in an epigrammatic mode so as to invent a freer metrics without yielding to "free-versism ". The last part of the paper consists in delineating the various expressions of theatricality and its relation to poetics in this collection of poems.

Mots-clés : Lorca - Cante Jondo - Poétique - Métaphore - Copla - Métrique Théâtralité.

Doema DEL Cante Jondo ${ }^{1}$, livre écrit en 1921 par Lorca mais qui ne 1 sera publié que dix ans plus tard, est sans nul doute le recueil dont il conviendrait, plus que pour tout autre, de réévaluer l'importance dans la trajectoire de l'œuvre poétique lorquienne en analysant ses véritables objectifs et les moyens mis en œuvre pour les atteindre. Pour tenter de mener à bien une telle démarche, il faut se garder de tomber, comme l'indique Claude Esteban, dans certains topiques dont le premier serait " celui d'une poésie toute soumise aux invites ingouvernées de l'inspiration » pouvant "oblitérer l'itinéraire véritable et le lieu proprement poétique où se sont inscrits l'exigence et le travail d'écriture $»^{2}$. Il suffit, d'ailleurs, de se reporter aux différentes conférences lorquiennes, aux entretiens accordés par le poète et surtout à son abondante correspondance, pour comprendre que chacune de ses déclarations reflète, quel qu'en soit le mode, la volonté métapoétique d'un artiste lucide, conscient de sa poétique et qui à travers l'attention conférée aux mots, aux images, aux mètres et à leur prosodie essaye de la cerner au plus près tout en sachant qu'il lui est en réalité impossible de la définir définitivement ${ }^{3}$.

Mon intention, bien évidemment, n'est pas d'ignorer les emprunts, sans cesse remaniés et réactualisés, au " chant primitif andalou » et les nombreuses

1. F. García Lorca, Poema del Cante Jondo - Romancero gitano, edición de Allen Josephs y Juan Caballero, Madrid, Cátedra, 1994. Tous les vers cités issus de ces deux livres seront suivis des références propres à cette édition.

2. "Architectures de l'obscur", préface à Romancero gitan, Poème du chant profond, de F. García Lorca, traduit par Claude Esteban, Paris, Aubier, bilingue, 1995, p. 8.

3. Cette impossibilité à " définir définitivement » sa poésie, assortie d'une extrême lucidité quant à ce en quoi doit consister un poème, est clairement exprimée par Lorca quand il déclare à Gerardo Diego, dans la "Poética " (de viva voz) incluse dans la célèbre anthologie Poesía española contemporánea (1901-1934): « Ni tú ni yo ni ningún poeta sabemos lo que es la poesía. [...]. Si es verdad que soy poeta por la gracia de Dios -o del demonio-, también lo es que lo soy por la gracia de la técnica y del esfuerzo de darme cuenta en absoluto de lo que es un poema ». Signo, Madrid, 1934, Taurus, 7a edición, 1974, p. 403. 
références liées à ce dernier, mais lire ce recueil à la lumière exclusive ou abusive de la tradition jonda, voire de ce que Lorca écrira plus tard à propos $\mathrm{du}$ duende, pourrait faire oublier les véritables visées de ce livre, porteur d'une poétique ambitieuse qui apparaît à un certain moment pour répondre aux questionnements d'un poète, encore au commencement de son œuvre, et qui, connaisseur averti de toute une tradition populaire et savante, n'en est pas moins ouvert aux courants artistiques et poétiques les plus récents.

1921, l'année où fut écrit pour l'essentiel et en quinze jours seulement Poema del Cante Jondo, est aussi celle de la publication du premier recueil lorquien, Libro de poemas. Poema del Cante Jondo peut donc être considéré comme le deuxième recueil écrit par le poète, bien que, parallèlement à l'écriture de celui-ci, Lorca en écrive trois autres : Suites, Canciones et Primeras canciones qui, eux aussi, connaittront des publications plus ou moins tardives. La dernière, celle de Suites, ne sera possible que plus d'un demi siècle plus tard grâce aux travaux d'André Belamich ${ }^{4}$ qui l'exhumera des poèmes inédits et de quelques autres textes tirés de Primeras canciones. Les titres de ces recueils font tous référence à la musique et au chant, ce que confirment non seulement, souvent, les thèmes abordés mais également l'importance accordée par Lorca, poète pianiste et guitariste, à l'aspect phonique et prosodique des textes qui les composent.

Si Libro depoemas est le recueil lorquien le plus volumineux, il est également celui où domine le plus largement un locuteur personnel qui plus jamais ne s'imposera, dans la suite de l'œuvre, avec une telle prépondérance. Sur les 68 poèmes du recueil, 42 emploient une voix poématique qui a recours, parfois de façon très répétée au sein d'un même poème, à un pronom de première personne, très souvent en position de sujet. Cette tendance reflète à l'évidence - malgré la qualité d'un recueil qui constitue une véritable mine pour les métaphores lorquiennes à venir et qui consigne déjà les premiers signes destructeurs propres au monde lorquien - la présence d'un Moi effusif et sentimental encore proche d'un héritage romantique, symboliste et moderniste. Or, ce qui caractérise l'écriture des recueils suivants c'est l'éradication de cette présence effusive et par trop sentimentale du Moi. Cette éradication est possible en ayant recours à une forme souvent très condensée qui semble s'organiser autour d'une image devant fonctionner alors comme un chiffre et dont la force, comme dans les haïkus ou les greguerias ramoniennes réside dans l'extrême concentration et concision. C'est ainsi que certains poèmes de Suites ou de Canciones ne comptent souvent que

4. Pour la reconstitution des Suites, cf. Federico García Lorca, Euvres complètes, tome I, édition d'André Belamich, Paris, Pléiade, 1981, p. 1270-1273. 
4 ou 6 vers, ou visent la fulgurance énigmatique d'une métaphore à la fois inquiétante et humoristique tel le distique qui clôt "Susto en el comedor» :

\section{(Grulla dormida, la tarde puso en tierra la otra pata).}

À cette ascèse langagière s'ajoutent parfois, comme dans le poème "Cazador" de Canciones, des jeux d'oppositions soulignés par des parallélismes et l'ellipse d'un personnage ou de son action puisqu'en dehors $\mathrm{du}$ titre, le chasseur responsable de la mort des oiseaux aperçus en plein vol (;Alto pinar!l Cuatro palomas por el aire van) puis gisant sur le sol (;Bajo pinar!/ Cuatro palomas en la tierra están.) est absent du poème, ne pouvant s'y glisser que de façon implicite et métonymique. Car entre les vers précités qui ouvrent et referment ce court poème, s'insèrent quatre pentasyllabes pour suggérer la mort causée par le chasseur à l'affût en attribuant par hypallage aux ombres des oiseaux la blessure mortelle à venir (Cuatro palomas/ vuelan y tornan/ llevan heridas/ sus cuatro sombras); une mort tragique qui prend effet lorsque les palombes tombent sur le sol, là même où les attendaient leurs ombres. De tels poèmes montrent la volonté de se débarrasser de ce qui est anecdotique pour se concentrer sur la force de l'image. Le court poème "Cazador " se présente comme un petit tableau stylisé, un minuscule triptyque (vol/ombres/mort) qui suggère sans la nommer cette idée de mort tragique qui domine le langage lorquien. Mais ce qui attire l'attention dans ce poème c'est que les formes s'abrègent jusqu'à l'épure pour faire ressortir l'antithèse vie-mort sans insister sur les conséquences possibles de la destruction. D'une certaine façon un poème comme celui-ci fonctionne comme un proverbe prononcé par une voix dont nous ignorons l'identité comme si cette voix était celle du texte lui-même qui n'est là que pour être dit. Après les épanchements adolescents et effusifs de Libro de poemas, Lorca semble concentrer le poème sur la force de l'image poétique qui n'est plus désormais instrumentalisée et sur le souci de trouver une voix plus vaste, moins soustraite au carcan circulaire d'un moi replié sur lui-même.

Pour Lorca la conception de l'image poétique trouve chez Góngora un maître et un modèle. Mais cet intérêt pour l'image est aussi ce qui mobilise toutes les avant-gardes des années 20, du Surréalisme à l'Ultraïsme. Cependant, s'intéresser à la tradition populaire eût semblé totalement contradictoire à n'importe quel artiste avant-gardiste de l'époque, futuriste, dadaïste, surréaliste, ultraïste, ou créationniste... Or, l'originalité et la particularité de Lorca c'est sa grande capacité à faire coexister des traits de ce qui est traditionnel avec ce qui est avant-gardiste tout comme il le fait entre 
ce qui est populaire et ce qui est savant. Ce syncrétisme va nourrir sa poétique tout au long de son œuvre, bien que celle-ci ne cesse pourtant d'évoluer. Par ailleurs, n'oublions pas que Lorca nous rappelle dans sa conférence gongorine que : en Andalucía la imagen popular llega a extremos de fuerza y sensibilidad maravillosa y las transformaciones son completamente gongorinas ${ }^{5}$. Cette observation montre clairement que pour lui poésie savante et poésie populaire ne sont pas antagoniques car elles peuvent avoir recours aux mêmes procédés. L'attachement de Lorca à la culture populaire - avec laquelle il se familiarise dès son enfance vécue dans la vega grenadine, en particulier, grâce à ces canciones de cuna que sont les nanas infantiles - se manifeste à travers son intérêt pour les Cancioneros dont il connaît un grand nombre par cœur, et pour la musique qui va l'amener à se lier d'amitié avec Manuel de Falla. De sorte que si la Residencia de estudiantes, où il séjourne régulièrement, s'ouvre aux nouveaux courants littéraires - Aragon y prononce à l'époque une conférence -, il n'est pas rare de trouver le poète chantant et jouant pour ses camarades de vieilles chansons populaires harmonisées par lui.

On peut même penser que la culture traditionnelle et populaire lui proposa très tôt une certaine poétique où venaient s'unir, par le biais de la musique, le lyrique et le dramatique sans tomber dans les pièges d'un Moi lié à une poésie effusive et sentimentale. On n'a peut-être pas assez réfléchi sur le fait que très souvent dans la tradition populaire la voix du chant soit anonyme : c'est le cas dans le Cante jondo et dans le Romancero viejo, deux traditions qui servent de substrat à deux livres lorquiens importants. Or, cet anonymat ne peut-il suggérer non la voix du peuple, comme se plaisaient à le croire les romantiques, mais celle du texte lui-même, comme quintessence à la fois de l'humain et du chant ? En s'éloignant d'un locuteur personnel omniprésent, la voix poématique lorquienne, bien qu'il s'agisse en réalité d'une poésie savante, semble s'engager avec le Cante Jondo et plus tard avec le Romancero gitano dans un cadre apparemment traditionnel et populaire, pour exprimer non ce qui est pittoresque - Lorca se défend de toute tendance costumbrista ${ }^{6}$ - mais ce qu'il y a de plus universel et de façon

5. "La imagen poética de don Luis de Góngora », in Federico García Lorca, Obras completas, tomo III, edición de Arturo del Hoyo, Madrid, Aguilar. Tous les extraits des conférences, des entretiens et de la correspondance seront cités dans cette édition. À propos de cette conférence gongorine, on pourra consulter l'analyse qui en est faite par Nadine Ly, "Lorca y la teoría de la escritura: "La imagen poética de don Luis de Góngora" ", in Valoración actual de la obra de García Lorca, Madrid, Casa de Velázquez, 1988.

6. C'est ainsi que profondément exaspéré par l'incompréhension régnant autour du Romancero gitano, qui veut faire de lui un poète populaire et l'enfermer dans le " gitanisme ", il écrit, en janvier 1927, à Jorge Guillén : " Me va molestando un poco mi mito de gitanería. Confunden mi vida y mi carácter. Los gitanos son un tema. Y nada más. Yo podría ser lo 
à ce que l'anecdote puisse céder le pas au mythe. De sorte que le modèle d'une voix anonyme provenant de la tradition populaire - c'est le cas du Cante jondo - convient parfaitement à la voix poématique lorquienne qui, à son tour, aspire à être anonyme et universelle bien qu' elle s'exprime à travers une poésie savante dont l'auteur, lui, est connu de tous.

Afin de cerner au mieux les enjeux de Poema del Cante jondo, nous nous proposons donc de voir ce qui a pu intéresser Lorca dans le chant primitif andalou, comment il a subtilement structuré son livre en diverses sections et comment au sein de celles-ci le texte se fonde sur la force de l'image dans des poèmes dont la forme inspirée du Cante jondo est en fait totalement réinventée pour servir une véritable poétique de l'épure, pleine de modernité mais où se profile déjà, comme nous le constaterons, un habile mariage entre poésie et théâtralité.

\section{LE CHANT PRIMITIF ANDALOU}

Soyons clair, ce qui m'importe ici ce n'est pas tant le Cante jondo, ce qu'il est ou ce qu'il fut réellement que la façon dont il est perçu par Lorca, aidé en cela, il est vrai, par le compositeur Manuel de Falla. Qu'est-ce qui a pu intéresser Lorca dans le Cante Jondo et comment l'a-t-il assimilé pour écrire Poema del Cante Jondo ? N'oublions pas d'emblée que le mot Poema qui ouvre le titre du recueil indique clairement qu'il s'agit avant tout d'une création poétique originale. Pour savoir comment Lorca a perçu le Cante Jondo et comment celui-ci a pu lui servir pour écrire son livre, nous disposons de deux conférences consacrées à ce sujet : El cante jondo. -Primitivo canto andaluz et Arquitectura del Cante jondo, bien qu'il soit plus approprié de parler d'une seule car la seconde conférence n'est en fait qu'une refonte de la première, dix ans plus tard, avec peu d'éléments nouveaux, excepté une réflexion sur le rôle joué par la guitare. C'est dans la nuit du 19 février 1922 que Lorca prononce sa première conférence, El cante jondo. -Primitivo canto andaluz au centre artistique de Grenade. Cette conférence est destinée à préparer un concours de Cante Jondo qui doit avoir lieu les 13 et 14 juin 1922 à Grenade. À la même époque nous savons que Lorca prend des cours de guitare flamenca avec deux gitans de la Vega et que sa conférence réutilise certaines parties d'une brochure publiée par les organisateurs et composée d'après des notes rédigées par Falla sur le Cante Jondo. D'emblée, le poète

mismo poeta de agujas de coser o de paisajes hidráulicos. Además el gitanismo me da un tono de incultura, de falta de educación y de poeta salvaje que tú sabes que no soy. No quiero que me encasillen ». " Prosa-Cartas ", Op. cit., p. 902. 
distingue le Cante Jondo et le Flamenco qui, selon lui, est une contrefaçon du premier. Le Cante demeure à ses yeux le seul primitivo canto andaluz, maravillosa verdad artística, chant millénaire teñido por el color misterioso de las primeras edades ${ }^{7}$ qui, contrairement au Flamenco, n'a rien à voir avec des cosas inmorales, la taberna, la juerga, el tablado de café, el ridículo jipio, ; la españolada en suma! ${ }^{8}$. Lorca nous explique que, contrairement au Flamenco qui est un chant relativement moderne (il ne prend sa forme définitive qu'au XVIII ${ }^{\mathrm{e}}$ siècle), le Cante Jondo est un balbuceo qui remonte non seulement aux primitivos sistemas musicales de la India mais à las primeras manifestaciones del canto ${ }^{9}$.

De sorte que pour le poète el Cante Jondo se acerca al trino del pájaro, al canto del gallo y a las músicas naturales del bosque y de la fuente ${ }^{10}$. D'où il déduit qu'il s'agit du chant primitif le plus ancien de toute l'Europe et qu'il porte en sus notas la desnuda y escalofríante emoción de las primeras razas orientales. À travers les images de ce discours historico-mythique, Lorca veut suggérer que le chant primitif andalou pourrait avoir précédé la parole, qu'il est un cri originel, à la fois pur et pathétique, qui conserve les traces de temps immémoriaux. C'est ce même cri fondateur du Cante Jondo qui donne lieu aux poèmes «El grito » et " $i A y !$ " (p. 148 et 158). De sorte que le qualificatif Jondo - prononciation andalouse et gutturale de hondo - est, selon Lorca, tout à fait approprié car ce chant est hondo, verdaderamente hondo, más que todos los pozos y los mares que rodean el mundo, mucho más hondo que el corazón actual que lo crea y la voz que lo canta, porque es casi infinito ${ }^{11}$.

Pour asseoir avec plus d'autorité son discours en grande partie mythique, le poète recourt à l'autorité du maître Falla, qui a analysé les origines et l'évolution historique de ce chant ainsi que sa structure musicale. En suivant l'analyse de Manuel de Falla, Lorca identifie les trois faits qui, selon le compositeur, intervinrent dans la création du Cante jondo : l'adoption par l'église espagnole du chant liturgique byzantin, l'invasion arabe de 711 et l'arrivée au XVe siècle des gitans - estas gentes misteriosas y errantes - qui vont lui donner sa forme définitive. Cependant, cela ne signifie pas pour autant que ce chant leur appartienne en propre, car s'il existe des gitans en d'autres lieux, seuls les gitans andalous le cultivent, d'où Lorca conclut que ce chant

7. «El Cante Jondo, Primitivo canto andaluz », op. cit., p. 197.

8. Ibid., p. 195.

9. Ibid., p. 197.

10. Ibid., p. 197.

11. Ibid., p. 204. 
existia en germen en esta región ${ }^{12}$. Le poète est avant tout soucieux d'affirmer l'origine immémoriale de ce chant qui, pour lui, ne put se préserver qu'en Andalousie. En réalité, lorsqu'il prononce cette conférence, Lorca a déjà écrit l'essentiel de son Poema del Cante Jondo comme le laissent entendre les images d'une phrase, où en parlant de la siguiriya gitane, il déclare que celleci lui inspire un camino sin fin... el camino donde murió el primer pájaro y se llenó de herrumbre la primera flecha ${ }^{13}$. Ces images font clairement allusion à celles employées dans son poème "La guitarra ": Llora flecha sin blanco,/ la tarde sin mañana, / y el primer pájaro muerto. (p. 147).

Il est important de comprendre qu'en choisissant le Cante Jondo comme source d'inspiration, Lorca ou plutôt le locuteur lorquien cesse de proclamer une douleur individuelle pour incarner la Pena, le malheur collectif qui n'est même pas seulement andalou mais universel. Il est d'ailleurs intéressant d'observer qu'en présentant le Cante Jondo, Lorca, d'une certaine façon, en vient à gloser ses propres poèmes d'inspiration jonda. Si bien que lorsqu'il rappelle que battent dans les tercets et les quatrains de la seguiriya et de ses dérivés las más inifinitas gradaciones del Dolor y la Pena, puestas al servicio de la expresión más pura y exacta ${ }^{14}$, il ne fait que signaler ce vers quoi tendent ses propres poèmes : "une ambiance " et "une justesse émotionnelle" exprimée avec la plus grande «stylisation ». Dans le chant primitif andalou Lorca trouve le modèle d'une épure formelle dans laquelle il peut condenser avec la plus grande efficacité possible et avec un sens tragique de la vie des images fortement lyriques et dramatiques. À propos des coplas du Cante Jondo, il nous dit qu'elles ont un fondo común: el Amor y la Muerte [...], pero un amor y una muerte vistos a través de la Sibila, ese personaje tan oriental, verdadera esfinge de Andalucía ${ }^{15}$. Cette observation nous suggère que ces vers mélancoliques constituent de petites énigmes où, comme ajoute le poète, late la pregunta, pero la terrible pregunta que no tiene contestación. Lorca fait ressortir dans le chant primitif andalou la ausencia de medio tono, car il est, selon lui, dépourvu "d'humbles états d'âme et de sentiments ingénus". C'est un chant pathétique qui o grita a las estrellas o besa el polvo rojizo de los caminos ${ }^{16}$ mais toujours chanté dans la nuit, ce qui nous renvoie au décor essentiellement nocturne de son Poema del Cante Jondo et même à celui du Romancero gitano, où sur les 18 romances, seul le Romance de la monja gitana évoque une situation diurne. Lorca explique enfin comment dans ce chant

12. "Arquitectura del Cante Jondo ", p. 219.

13. "El cante jondo, Primitivo canto andaluz », p. 198.

14. Ibid., p. 205.

15. Ibid.

16. Ibid., p. 206. 
andalou tout est cosmique et panthéiste en déclarant que todos los poemas del cante jondo son de un magnifico panteismo, consultan al aire, a la tierra, al mar, a la luna et qu'en eux la mujer se llama Pena ${ }^{17}$. De sorte que Lorca précise que todos los objetos exteriores toman una aguda personalidad y llegan a plasmarse hasta tomar parte activa en la acción lírica ${ }^{18}$. Cette phrase de la conférence, si nous ouvrons le recueil lorquien, convient à la perfection pour chacun de ces objetos exteriores que proposent des poèmes comme " $\mathrm{La}$ guitarra ", "Candil ", "Chumbera ", "Pita " ou "Cruz ». Par ailleurs, cette même conférence sur le primitif chant andalou montre comment les objets, les éléments de la nature ou un sentiment comme la Pena deviennent, grâce à la prosopopée, des interlocuteurs privilégiés de la voix qui chante. C'est ainsi que le poète écrit : En las coplas la Pena se hace carne, toma forma humana y se acusa con una línea definida. Es una mujer morena que quiere cazar pájaros con redes de viento ${ }^{19}$.

La prosopopée est un procédé constamment utilisé par Lorca dans sa poésie et plus particulièrement dans son Poema del Cante Jondo pour personnifier les formes poétiques et musicales que sont la siguiriya, la soleá ou la petenera, souvent représentées comme des danseuses assimilées aux cordes mêmes de la guitare. Et quand Lorca rappelle que dans le Cante Jondo "le vent est un personnage " qui " apparaît comme un géant ", il est difficile de ne pas penser au personnage du viento-hombrón, ce vent lubrique qui harcèlera Preciosa dans "Preciosa y el aire ", romance écrit quelques années plus tard.

Même certains éléments de la conférence apparemment plus techniques semblent justifier quelques aspects stylistiques et formels de Poema del Cante Jondo. C'est le cas de l'enharmonismo como medio modulante appliqué, selon Lorca, au Cante Jondo dont le cadre mélodique dépasse rarement les limites d'une sixte réitérant de façon obsédante el uso de una misma nota. Pour Lorca ce procedimiento propio de ciertas fórmulas de encantamiento produit la impresión de una prosa cantada, destruyendo toda sensación de ritmo métrico, aunque en realidad son tercetos o cuartetos asonantados sus poemas ${ }^{20}$. En effet, la siguiriya gitane à laquelle se réfère tant le poète correspond en réalité, comme nous le verrons, à un schéma métrique assez précis, mais Lorca dans son recueil va l'interpréter à sa façon avec un vers fluctuant capable de produire "l'impression d'une prose chantée sans sensation de rythme métrique », bien que celui-ci existe.

17. Ibid., p. 209.

18. Ibid.

19. Ibid.

20. «Arquitectura del Cante Jondo », p. 219-220. 
Rappelons, pour conclure sur le chant primitif andalou, qu'il est important de ne pas confondre une poésie inspirée par le Cante Jondo - celle écrite par Lorca - avec la poésie propre au Cante Jondo - celle qui appartient à la tradition. Car comme l'indique Franciso Rubio Morales, «ce qui intéresse Lorca ce sont les arcanes et les énigmes qu'il pressent dans le Cante " et à partir desquels "il poétise et sublime " ${ }^{21}$ jusqu’à atteindre la représentation mythique de l'Andalousie et de son chant primitif, mais, avec une expression, bien évidemment différente du Cante Jondo originel et traditionnel. Le Cante Jondo reste autonome car il n'est pour Lorca qu'une source d'inspiration. On peut, d'ailleurs, observer que le propre poète ironise à propos de ceux qui essaient d'imiter ou de contrefaire le Cante Jondo, lorsqu'il déclare : iQué diferencia tan notable entre los versos de estos poetas - Manuel Machado qui écrivit un Cante hondo est clairement visé - y los que el pueblo crea! 'La diferencia que hay entre una rosa de papel y otra natural! C'est pourquoi Lorca, pensant certainement à son propre travail poétique, donne le conseil suivant : Se debe tomar del pueblo nada más que sus últimas esencias y algún que otro trino colorista, pero nunca querer imitar fielmente sus modulaciones inefables, porque no hacemos más que enturbiarlas ${ }^{22}$. Le message est des plus clairs, laissant entendre qu'en écrivant Poema del Cante Jondo, Lorca ne s'est pas livré à une imitation mais bien à une recréation originale.

Mais une recréation destinée à le faire avancer dans sa propre poétique, autrement dit dans ses propres questionnements et réponses aussi bien ontologiques qu'esthétiques. C'est pourquoi certaines phrases de la conférence consacrée au chant primitif andalou peuvent presque résonner, pour qui sait les écouter, comme celles d'un véritable manifeste poétique. Cela est particulièrement patent lorsque Lorca nous apparaît, au milieu de sa conférence, comme un jeune poète moderne hanté par la quête d'une expression poétique succincte, concise, libre de tout excès rhétorique qui trouve, à ce moment-là, dans le Cante Jondo un modèle qui, bien qu'il appartienne à la tradition, se prête idéalement à la modernité. C'est bien ce qu'exprime cette phrase "générationnelle ": Todos los poetas que actualmente nos ocupamos, en más o menos escala, en la poda y cuidado del demasiado frondoso árbol lírico que nos abandonaron los románticos y postrománticos quedamos asombrados ante los dichos versos ${ }^{23}$. Car "l'élagage du touffu arbre lyrique des romantiques et des postromantiques " est une tâche exigeante qui, à travers son souci de rigueur, semble englober aussi bien les orientations des

21. Federico García Lorca y el cante jondo, Valencia, Eutopías, 228, éd. Episteme, 1999, p. 10.

22. «El Cante Jondo - Primitivo canto andaluz », p. 208.

23. Ibid., p. 205. 
poètes de la future génération de 27 que celles des poètes qui tendent vers la " poésie pure " comme Valéry, voire Mallarmé, poète apparemment aux antipodes de l'écrivain andalou.

\section{L'ARCHITECTURE DE POEMA DEL CANTE JONDO}

Ce qui attire d'emblée l'attention dans le titre de ce livre, c'est qu'il a recours à un syntagme paradigmatique qui, après le poème-prélude, intitulé "Baladilla de los tres ríos", revient dans le titre de trois des quatre sections majeures qui servent de fondations au livre puisque nous avons successivement : Poema de la siguiriya gitana, Poema de la soleá et Poema de la saeta. Qu'il s'agisse du titre du recueil ou de celui de l'une des trois premières sections, nous avons, à chaque fois, un syntagme syntaxiquement identique : un même substantif, Poema qui désigne le genre, suivi d'un génitif qui se réfère à un mode d'expression général ou particulier du Cante Jondo. On peut observer qu'apparaît toujours le singulier Poema et non Poemas, comme c'était le cas dans le premier recueil lorquien, intitulé Libro de poemas, comme si ce qui prévalait ici était l'unicité ou l'unité. Comme si l'idée de " collectage » - recueil ou livre de poèmes - disparaissait devant celle d'unité considérée comme entité, c'est-à-dire comme essence d'un genre, celle du genre " poème ». Le singulier supprime le circonstanciel, le contingent pour insister avant tout sur l'essentiel, sur un travail poétique qui doit s'incarner devant le lecteur, durant le temps de sa lecture, transformant ce dernier en témoin de la création poétique. Chacun des quatre titres suggère, grâce au génitif, que l'origine du texte poétique lorquien est le Cante Jondo ou ses différentes modalités musicales (Siguiriya, soleá et saeta). Cela montre d'emblée quelle est la source d'inspiration du livre, lequel ne doit pas être considéré comme une imitation ou une reproduction, comme nous l'avons déjà signalé, mais comme une recréation originale, personnelle et libre du Cante Jondo. Car ce qui s'impose, c'est le Poema, autrement dit le texte poétique élaboré par Lorca, un texte totalement neuf qui utilise une tradition musicale et poétique populaire déjà existante et même séculaire comme point de départ mais pour donner immédiatement forme à un poème original, créé par un poète savant, Lorca. Le Cante Jondo se transforme ici en poème, celui-ci est son incarnation poétique. De sorte que ce que nous annonce par quatre fois ce syntagme paradigmatique (Poema de + genre musical andalou), c'est la façon dont la musique devient une image poétique, ce qui suppose bien évidemment une image polysensorielle, c'est-à-dire visuelle, émotive, sonore et rythmique. 
Le mot Gráfico - mot qui a des résonances plus avant-gardistes que jondas - remplace celui de Poema dans le paradigme qui sert de titre à la quatrième section (Gráfico de la petenera), substitution due probablement au fait que la petenera ne soit pas un chant aussi authentique et aussi jondo que ceux qui le précèdent parce que plus récent et plus flamenco. Ce mot de Gráfico connote une idée d'image visuelle qui par la suite se prolonge avec les portraits opposés et en vis-à-vis de Dos muchachas, titre de la première des quatre sections mineures, puis qui se confirme avec le titre de la seconde section mineure, Viñetas flamencas.

Bien que le recueil ait été écrit pour l'essentiel d'une seule traite, en une quinzaine de jours, en novembre 1921, et sans avoir connu ensuite de modification majeure avant d'être publié dix ans plus tard, sa structure très cohérente et équilibrée, qui vit néanmoins la mise à l'écart de 17 poèmes, mérite qu'on s'y arrête.

Le livre s'ouvre donc sur le poème "Baladilla de los tres ríos ", véritable prologue ou prélude qui sert d'introduction géographique et thématique au recueil. Les toponymes désignent deux villes d'Andalousie, Séville et Grenade avec leurs fleuves respectifs, le Guadalquivir pour la première et le Dauro et le Genil pour la seconde. D'emblée, le territoire du Cante Jondo est délimité : depuis la Sierra Nevada grenadine dont les rios bajan de la nieve jusqu'à l'embouchure sévillane du Guadalquivir. C'est ainsi que les trois fleuves, tout en traçant les limites géographiques de ce territoire jondo, suggèrent une obsession thématique de l'eau désireuse d'atteindre sa destination ultime : la mer, bien que seul le Guadalquivir puisse y aspirer car les deux autres appartiennent à une Grenade isolée, éloignée à jamais de la mer et condamnée à la frustration. Dans ce poème les chemins de l'eau nous mènent vers le centre émotionnel de l'Andalousie et nous annoncent déjà que chaque ville aura, au sein du livre, sa place et son heure de gloire. Grenade et Séville qui en sont bien évidemment les villes les plus importantes se manifesteront dans l'une ou l'autre des sections. De sorte que si Grenade est, avec Cordoue - non nommée dans ce poème -, une terre de siguiriya et de soleá - fondement de la première et de la deuxième section -, Séville, en revanche, est la ville de la saeta qui blesse avec amour et qui sert de cadre à la troisième section, laquelle précède la quatrième, consacrée, elle, à la petenera, et chargée d'évoquer la posibilité d'un camino étranger à ceux présentés antérieurement : Ni a Córdoba ni a Sevillal llegarán./ Ni a Granada la que suspira / por el mar (in "Camino", p. 176).

Quant au double distique de cette ballade-prélude (; Ay, amor/ que se fue y no vino ou i $A y$, amor que se fue por el aire!) qui à la façon d'un refrain surgit, comme un cri ou une plainte, entre les cuartetas ou coplas, il se 
manifeste comme une lamentation déjà assimilée à la Pena, lamentation sur la disparition de quelque chose de mystérieux qui est l'amour.

À la suite de ce poème-prologue - présentation de toute une géographie physique et lyrique où Lorca va mettre en scène sa propre vision du Cante -, nous découvrons une structure parfaitement cohérente et équilibrée ${ }^{24}$, constituée par une sorte de triptyque où sont combinés de façon très subtile le pair et l'impair afin de créer certains parallélismes :

- I) quatre sections majeures consacrées respectivement et successivement à la siguiriya gitane, à la soleá, à la saeta et à la petenera.

- II) quatre sections mineures où se mêlent le jondo et le flamenco, déjà introduit par la petenera, (puisque Lorca nous rappelle que celle-ci no cabe dentro de lo que normalmente se considera como cante jondo): "Dos muchachas", "Vinetas flamencas", "Tres ciudades", "Seis caprichos".

- III) et pour conclure deux dialogues qui sont deux courtes scènes de théâtre, suivies chacune d'une chanson : "Escena del teniente coronel de la guardia civil » avec "Canción del gitano apaleado » puis « Diálogo del Amargo " avec "Canción de la madre de Amargo ". Grâce aux Canciones, on renoue avec le chiffre 4 tandis que se manifeste de façon évidente le lien étroit qui lie le dramatique (les Diálogos) avec le lyrique (les Canciones) qui bientôt fusionneront grâce au style du Romance, employé dans Romancero gitano.

On peut observer que les quatre sections majeures qui constituent les fondations du Poème-livre ont chacune des dimensions analogues, car elles comptent respectivement 7, 10, 8 et 8 poèmes. Il convient aussi d'observer que chacune de ces sections semble constituer une histoire à part qui donne l'impression de progresser d'un poème à l'autre. Et dans chaque section l'apparition de la forme poétique et musicale, presque toujours personnifiée, se manifeste plus ou moins au même endroit au sein de chaque histoire. C'est ainsi que

- la siguiriya apparaît dans le $5^{\mathrm{e}}$ poème «El paso de la siguiriya » quand il en reste deux,

- la soleá surgit dans le $7^{\mathrm{e}}$ poème « La soleá " quand il en reste trois.

- la saeta apparaît dans le $6^{\mathrm{e}}$ poème ("Saeta ") quand il n'en reste que deux.

- et la petenera fait irruption dans le $5^{\mathrm{e}}$ poème ("Muerte de la Petenera ») quand il en manque trois pour que s'achève la section.

24. Nous ne nous intéressons ici qu'à la structure définitive du recueil ; pour ce qui est des rapports entre la genèse du livre et sa structure, il sera bon de se référer à la précieuse étude de Christian de Paepe qui précède son édition critique du livre, Federico García Lorca, Poema del Cante Jondo, Madrid, Espasa-Calpe, 1986, p. 41-100. 
Par ailleurs, il est intéressant d'observer que chacune de ces quatre sections ou histoires semble suivre un schéma et une progression relativement semblables :

- a) un début qui coïncide avec une vision panoramique, un paysage, une atmosphère et un décor

- b) une plainte ou un cri

-c) un silence

- d) l'apparition de la forme poétique et musicale

- e) l'éloignement de cette figure poétique et musicale

$-\mathrm{f}$ ) une nouvelle vision panoramique.

Les parallélismes entre une section et une autre sont évidents comme le laisse entendre le titre de certains poèmes comme par exemple, "El grito " de Poema de la siguiriya gitana, auquel fait écho le " ; Ay!" de Poema de la soleá ou comme le poème "Alba " qui termine la $2^{\text {ème }}$ section auquel semble répondre le poème "Madrugada " qui conclut, lui, la $3^{\text {ème }}$ section. Mais chaque section propose sa propre histoire qui est une des variations jondas de la Pena andaluza. C'est ainsi que dans le Poema de la soleá on peut suivre l'histoire de la mort énigmatique d'un inconnu poignardé dans la rue, écho d'autres douleurs, depuis le présage du fait-divers avec la présence dans différents poèmes du mot puñal (in "Puñal », "Encrucijada », "Sorpresa ») jusqu'à ses conséquences en passant par la mort et son deuil en la personne de la soleá. Une mort et une "Peine " qui acquièrent une dimension mythique pouvant s'apprécier de diverses façons.

Dans la $1^{\text {ire }}$ section, Poema de la siguiriya gitana, nous assistons à un récit allégorique de la siguiriya où domine la musique. La construction même de la section, quand nous observons les titres des poèmes, semble suivre l'ordre de la siguiriya gitane, rappelé par Lorca dans Arquitectura del cante jondo quand il dit : La siguiriya comienza por un grito terrible. Un grito que divide el paisaje en dos hemisferios iguales; después la voz se detiene para dejar paso a un silencio impresionante y medido [...] Después comienza la melodía ondulante e implacable ${ }^{25}$. Cette présentation coïncide parfaitement avec l'apparition de certains poèmes puisqu'après le poème "Paisaje ", nous avons le llanto du poème "La guitarra " (p. 146) - qui fait allusion au rasgueo qui amorce toute siguiriya -, suivi des poèmes "El grito " (p. 148) et "Silencio " (p. 149) avant de passer à «El paso de la siguiriya " (p. 150) qui correspond naturellement à la mélodie de ce chant pour conclure la section grâce à « $\mathrm{Y}$ después» (p. 152) avec les vers : Un ondulado/ desierto.

25. Op. cit., p. 218. 
La $2^{\text {ème }}$ de section, Poema de la soleá, comme nous l'avons déjà indiqué, est le récit d'une mort tragique et violente et de sa répercussion dans la rue et dans la campagne, qui entraîne l'arrivée de la soleá sous les traits d'une femme endeuillée et la réaction douloureuse des gitans (in "Cueva » p. 161).

La $3^{\text {ème }}$ section, Poema de la saeta, s'organise autour de la saeta, copla et prière andalouse, décochée comme une flèche, lorsque passent les statues de la Vierge et du Christ dans les processions de la Semaine Sainte à Séville. Mais, dans cette section, contrairement aux autres, la forme poétique et musicale, cette fois religieuse, ne semble pas personnifiée par une bailaora comme pour les formes profanes. En effet, le poème "Saeta " est dédié, comme l'exige la tradition, au Christ ou plutôt à sa représentation. Cependant, si nous sommes attentifs à l'histoire qui semble nous être contée dans cette section, nous découvrons la laïcisation de la saeta et voyons comment un personnage profane, la Lola, qui est une saetera, vient se substituer à l'image de la saeta personnifiée qu'on était en droit d'attendre. Car si nous prêtons attention aux diverses étapes de cette histoire énigmatique, nous voyons qu'elle commence d'abord avec l'arrivée, pour les processions, des saeteros, désignés comme arqueros, ce qui permet déjà de suggérer, grâce à l'allusion à l'arc - l'arme de Cupidon -, que l'amour religieux pourrait glisser vers l'amour profane. Ensuite, les poèmes semblent suivre un ordre presque liturgique ("Noche », "Procesión », "Paso », "Saeta ). Mais, après avoir constaté la non-personnification de la saeta - celle-ci aurait pu être blasphématoire ou trop éloignée de l'humain - surgit le poème "Balcón " (p. 172) avec le personnage d'une Lola saetera qui semble décocher des flèches d'amour sur les saeteros, lesquels au petit matin se retrouvent ciegos, autrement dit amoureux puisque le poème "Madrugada " (p. 173) conclut en disant : jAy, pero como el amor/ los saeteros/ están ciegos.

Quant à la $4^{\mathrm{e}}$ section, Gráfico de la petenera, si nous nous fixons sur le scénario imprécis mais néanmoins présent que semblent nous proposer les différents poèmes - c'est-à-dire sur leur ordre, leur évolution et la répétition de certains mots ou de certaines images -, il semble nous raconter l'histoire de cien jinetes. Une histoire constamment rythmée par la présence de la guitare. Ces cavaliers semblent, dès le début, porter le deuil anticipé de la Petenera, voire leur propre deuil, car, à l'affût, un certain maléfice prêt à frapper à tout instant entoure cette forme musicale, plus flamenca que jonda, et dont l'incarnation féminine est surnommée la perdición de los hombres. Et c'est ainsi que dans le poème "La muerte de la Petenera " (p. 179), la veillée funèbre de la défunte se conclut avec la mort des cien jinetes enlutados du poème "Camino " (p. 176) dont on ignorait alors la destination (Cien jinetes enlutados/ ¿Dónde irán,/ por el cielo yacentel del naranjal?). C'est pour 
cette raison que dans « La muerte de la petenera » se répète implacablement le refrain : cien jacas caracolean./ Sus jinetes están muertos. (p. 179). Or, le thème du cheval qui emporte son cavalier mort vers son destin est une image fréquente chez Lorca car il suffit de se rappeler les vers de "Canción de jinete " : Caballito negrol ¿Dónde llevas tu jinete muerto? Nous comprenons alors que el laberinto de las cruces où devaient achever leur course les cien jinetes endeuillés et déboussolés du poème "Camino" (p. 176) - Esos caballos soñolientos/ los llevarán, / al laberinto de las cruces - n'était autre que le cimetière avec ses multiples croix et allées. Les protagonistes semblent succomber, une fois de plus, à cause d'une passion sensuelle décrite comme cécité car ils ne conduisent ni ne dirigent leurs jacas, image de la passion dite sur le mode de l'animalité. En réalité, tout laisse supposer que ces cien jinetes lancés au galop étaient déjà potentiellement morts, car incapables de se soustraire à leur destin tragique. La logique poétique de cette section amène ensuite avec "Falseta" (p. 181), l'enterrement de cette femme de mauvaise vie que fut la petenera (Tu entierro no tuvo niñas/ buenas) puis dans le poème suivant, "De profundis " (p. 182), l'enterrement des cien jinetes, clairement désignés désormais comme des amoureux (Los cien enamorados/ duermen para siempre). Comme toujours l'histoire est énigmatique et par conséquent ouverte à différentes interprétations comme le laisse supposer l'amour passionné et tragique des cien jinetes dont le chiffre fonctionne comme une formule populaire et hyperbolique. Quant au dernier poème de cette $4^{\mathrm{e}}$ section, "Clamor» (p. 183), on peut observer qu'il se présente comme une nouvelle version plus étendue et plus élaborée du poème initial "Campana» (p. 174), ce qui ne doit pas nous surprendre puisque le mot clamor peut avoir le sens en espagnol de toque de campanas por los difuntos, autrement dit de "glas". Cette signification funèbre justifiée par les nombreuses morts antérieures est confirmée par le passage du singulier campana, dans le premier poème, au pluriel campanas utilisé à présent pour clore cette section. Une section dominée donc par le champ lexical du son avec un final crescendo et fortissimo qui ne s'éteint que pour laisser place au silence et au passage visuel des proras de plata.

Tout ce champ lexical du son et de la musique ne doit pas nous faire oublier, tout au long de cette section, le rôle fondamental joué par la guitare dès le sous-titre du poème "Campana ", qui n'est autre que "bordón ", mot désignant la corde la plus grosse et la plus grave de l'instrument. Une corde citée au vers 14 de «Muerte de la Petenera" (p. 180) : y el bordón de una guitarral se rompe tandis que les six cordes de la guitare servent de titre à un autre poème, "Las seis cuerdas" (p. 177), avant d'être métaphorisées comme les seis gitanas qui dansent dans le huerto de la Petenera (in "Danza ", 
p. 178). Quant à l'allégorie de la mort qui chante dans le dernier poème, "Clamor ", p. 183, elle s'accompagne, elle aussi, d'une guitare désignée alors comme vibuela blanca:

\author{
Por un camino va \\ la muerte, coronada, \\ de azahares marchitos. \\ Canta y canta \\ una canción \\ en su vihuela blanca, \\ y canta y canta y canta.
}

Car dans le negro aljibe de madera propre à la guitare ("Las seis cuerdas", p. 177), autrement dit dans sa caisse de résonance, flottent les rêves, les soupirs et «les sanglots des âmes perdues». De là l'omniprésence de cet instrument fondamental pour le Cante Jondo dans la $1^{\text {ère }}$ et la $4^{\text {ème }}$ section de ce premier volet. Comme l'indique Lorca dans Arquitectura del cante jondo, la guitare doit être un fondo para la voz y debe estar supeditada al que canta, au cantaor, bien que de la virtuosité du guitariste naisse, selon le poète, la falseta, - titre d'un des poèmes -, qui correspond à cette brève phrase mélodique, cette fioriture qui s'intercale entre les suites d'accords destinés à accompagner la copla. C'est pourquoi Lorca définit dans sa conférence la falseta comme el comentario de las cuerdas $^{26}$.

La $1^{\text {ère }}$ des quatre sections mineures, Dos muchachas, semble se concentrer sur le visuel, sur l'art du portrait, avec un diptyque fondé sur l'opposition entre la sensuelle et la frivole Lola et la chaste et sage Amparo. Un art du portrait qui se prolonge dans la $2^{\text {ème }}$ section mineure beaucoup moins jonda - dont le titre Viñetas flamencas fait allusion à un art graphique - avec les portraits qui servent d'hommage à trois cantaores de la fin du XIX ${ }^{\mathrm{e}}$ siècle ("Retrato de Silverio Franconetti ", "Juan Breva " et "Café cantante" qui évoque la cantaora La Parrala.). En revanche, les trois poèmes restant ("Lamentación de la muerte ", "Conjuro », « Memento ») renouent avec les thèmes des 4 sections majeures.

Ensuite, la section Tres ciudades inclut trois poèmes ("Malagueńa", «Barrio de Córdoba » et « Baile»). Avec « Malagueña » (p. 196), nous avons à nouveau la prosopopée d'une forme musicale, la malagueña identifiée à la ville de Málaga et qui permet de comprendre l'allégorie : La muertel entra y salel de la taberna comme le résultat de cette musique et de son chant sur le thème de la mort, qui commencent à s'entendre à l'intérieur du

26. Op. cit., p. 221. 
cabaret. Le second poème, « Barrio de Córdoba » (p. 197) présente la veillée funèbre d'une enfant, accompagnée par seis ruiseñores (que) la lloran en la reja, nouvelle allusion aux six cordes qui passent au-dessus de la rosace de la guitare, instrument auquel il est clairement fait allusion dans le distique conclusif : Las gentes van suspirandol con las guitarras abiertas. Quant au $3^{\text {e }}$ poème, "Baile " (p. 198), il présente, avec la danse obscène dans les rues de Séville de la vieille Carmen, - qui déjà semble annoncer la María Josefa de La casa de Bernarda Alba - le spectacle honteux et dangereux de l'Éros qui scandalise les mères bien pensantes.

$\mathrm{La} 4^{\mathrm{ème}}$ et dernière section, intitulée Seis caprichos, regroupe six poèmes aux métaphores à la fois gongorines et avant-gardistes et aux allusions mythologiques (Polyphème, Laocoon, Daphné, Atys) et ludiques, courts textes destinés à célébrer des objets et des plantes typiques du cadre ethnographique du Cante Jondo: la guitare, le candil, le crótalo (qui correspond aux castagnettes), la chumbera, la pita et la cruz. La cruz définie comme punto finall del camino vient clore de façon très cohérente la $4^{\mathrm{e}}$ section et d'une certaine manière Poema del Cante Jondo puisque la $5^{\mathrm{e}}$ section, fondée sur deux diálogos avec leurs canciones respectives, est un ajout postérieur de 1925 qui, cependant, permet grâce à son aspect fortement dramatique de mettre en rapport ce livre avec Romancero gitano.

\section{GÓNGORA OU UNE THÉORIE LORQUIENNE DE L'IMAGE POÉTIQUE}

Toutes les poétiques avant-gardistes européennes des années 20, futuristes, dadaïstes, surréalistes, ultraïstes et créationnistes conçoivent le texte poétique comme un réservoir d'images inédites et surprenantes, que ne peut exprimer le langage rebattu, conventionnel et fonctionnel de la communication quotidienne, ce que Mallarmé appelait avec mépris « la langue de l'universel reportage ». Ces poétiques ignorant souvent la ponctuation, modifiant parfois la typographie pour donner dans des cas extrêmes à leurs poèmes l'aspect d'objets comme les calligrammes d'Apollinaire, essaient de rompre l'ordre métrique et syntaxique ainsi que la continuité sémantique parce que leur seul souci est la production d'images nouvelles et inattendues. Le poétique, au nom souvent d'une liberté absolue, tend à s'identifier presque exclusivement avec l'aptitude à créer une profusion d'images inédites. Les échos de ces mouvements parviennent, bien sûr, jusqu’à la Residencia de estudiantes et si nous lisons certains vers propres à ces poétiques, ils peuvent parfois ne pas paraître si éloignés de certains vers lorquiens. C'est ainsi que l'ultraïste Pedro Garfias dans son poème "Peregrino" compose les vers 
suivants : Fluian horizontes de sus ojos/ Traia rumor de arenas en los dedos/ $Y$ un haz de sueños rotos/ Sobre sus hombros trémulos, tandis que Buñuel, grand ami de Lorca, éphémère poète, partisan des ultraïstes et admirateur des surréalistes écrit le vers : Un barco puede naufragar en una gota de mi sangre. À cette époque composer un poème, c'est donc avant tout aller à la recherche d'images insolites, qu'il s'agisse d'une méthode qui prétende recourir à une soi-disant irrationnalité, spontanée et révélatrice du subconscient ou d'une autre fondée sur une lucide et exigeante agudeza conceptiste. L'image poétique, comme le rappelle Charles Marcilly, est dans l'air du temps ${ }^{27}$.

C'est dans ce contexte et après celui d'une " poésie pure »-poésie cérébrale, partisane d'une certaine " asepsie " ou d'un " élagage lyrique ", héritière de Mallarmé et de Valéry dont l'admirateur est Jorge Guillén, un autre ami de Lorca - que le poète andalou compose son Poema del Cante Jondo. N'oublions pas non plus l'importance, en 1925, de La deshumanización del arte, cet essai très polémique de José Ortega y Gasset, qui va donner lieu à tant de malentendus à cause du concept de deshumanización, qui recommande un art cérébral, non soumis à l'effusion ni à la sentimentalité. Lorca, sans être un chef de file, apparaît très vite comme la figure la plus brillante d'un groupe de poètes qui, contrairement aux mouvements d'avant-garde, ne se considère pas comme une école ni ne publie de programme ou de manifeste. Ces poètes, lecteurs passionnés de textes poétiques appartenant parfois à une lointaine tradition n'en sont pas moins ouverts à la modernité, voire à l'avant-garde. Modernité et tradition ne leur semblent pas nécessairement antagoniques, si bien qu'en 1927, la célébration du tricentenaire de la mort de Góngora va donner lieu à une réhabilitation de ce poète baroque et hermétique du $\mathrm{XVII}^{\mathrm{e}}$ ainsi qu'à une profonde et profitable réflexion sur l'image poétique.

Comment ne pas penser que la célèbre conférence de Lorca, La imagen poética de don Luis de Góngora, prononcée en 1926 à l'Ateneo de Grenade n'est pas, en fait, l'expression d'une théorie lorquienne de la métaphore mise en ouvre dès Poema del Cante Jondo ? Une conférence qui est le fruit d'une réflexion solide et continue, comme le suggère une lettre adressée à Jorge Guillén où Lorca lui confie : La he trabajado tres meses ${ }^{28}$. Au début de sa conférence Lorca, s'adressant au public, laisse entendre à ce dernier qu'il ne peut ignorer ce qu'est une image poétique : Ya os supongo enterados de [...] (lo)

27. Dans son article «Historicidad de la imagen poética en la obra de Federico García Lorca ", in Valoración actual de la obra de García Lorca, Madrid, Casa de Velázquez, 1988, Charles Marcilly déclare : Cosa de ambiente, de aire del tiempo. Esto se daba en Madrid, en la Residencia de Estudiantes por los años veinte., p. 132. Les vers de Pedro Garfias et de Luis Buñuel que nous avons cités ont été relevés dans cet article.

28. "Carta a Jorge Guillén », op. cit., p. 884. 
que es una imagen poética. Todos habéis estudiado Preceptiva y Literatura ${ }^{29}$... Cette habile stratégie allusive-élusive lui permet d'emblée de maintenir une certaine imprécision quant à l'identité exacte de l'image poétique qui, bien qu'elle renvoie principalement à la métaphore, pourra se référer, par moments, à d'autres tropes plus ou moins proches ou complémentaires comme la comparaison, la synecdoque, la métonymie, la synesthésie, l'hypallage, l'oxymore, l'hyperbole, la prosopopée, l'allégorie, la dilogie... Si Lorca se refuse à employer ces termes rhétoriques, c'est pour ne pas s'enfermer dans des définitions trop réductrices. De sorte que lorsqu'il parle d'image poétique, il se réfere à quelque chose de plus vaste et de plus riche que ces termes et leurs définitions, afin de pouvoir unir savoir et invention et esquisser une image poétique qui soit la plus ouverte possible et où puissent parfois se combiner plusieurs tropes à la fois.

Cependant, il semble clair que, pour lui, l'image poétique est surtout synonyme de métaphore comme le suggère la définition qu'il nous propose : $Y$ una imagen poética es siempre una traslación de sentido ${ }^{30}$ car étymologiquement en grec metaphora signifie justement " transfert » ou " transposition ", celle d'un objet pour un autre objet, ou celle d'une idée pour une autre idée. Il rappelle avec des exemples précis et commentés, que "le langage est fait à base d'images " et que celles-ci abondent, tout particulièrement en Andalousie avec une subtilité et une sensibilité complètement gongorines. Après avoir disqualifié les vaines oppositions signalées par l'histoire littéraire entre les champions de la clarté (les llanistas) et ceux de l'obscurité comme Góngora (les tenebrosistas), il en vient, néanmoins, à préciser que Góngora est un poète qu'il faut aller chercher, et pour lequel la lecture ne peut se passer de l'étude. Pour Lorca, Góngora a accompli une véritable « révolution lyrique " car il a inventé por primera vez en castellano un nuevo método para cazar y plasmar las metáforas ${ }^{31}$. Et pour le prouver, il cite et analyse des images gongorines tirées des Soledades, qu'il s'agisse de métaphores par apposition entre comparé et comparant (Pintadas aves - citaras de pluma-) ou de figures qui confondent métonymies et synesthésies comme ces verdes voces qui désignent le chant des oiseaux dissimulés par de luxuriantes frondaisons ou d'autres synesthésies fondées sur des métonymies, des synecdoques et des métaphores pour exprimer le chant mélodieux de ces mêmes oiseaux : voz pintada, canto alado,/ órgano de pluma. Lorca qui pense autant à sa propre poésie qu'à celle du poète cordouan est convaincu que un

29. "La imagen poética de don Luis de Góngora », op. cit., p. 223.

30. Ibid., p. 224.

31. Ibid., p. 228. 
poeta tiene que ser profesor en los cinco sentidos corporales, en este orden: vista, tacto, oido, olfato y gusto. Et il ajoute que para poder ser dueño de las más bellas imágenes tiene que abrir puertas de comunicación entre todos ellos y con mucha frecuencia ha de superponer sus sensaciones y aun disfrazar sus naturalezas ${ }^{32}$. Pour lui, le poète chasseur d'images doit donc recourir à tous ses sens et les exacerber en fonction d'un certain ordre (vue, toucher, ouïe, odorat et goût), en les faisant communiquer les uns avec les autres, un peu comme dans la théorie baudelairienne des correspondances. D'où de constantes remarques synesthésiques qui doivent susciter la création d'une image polysensorielle bien que le point de départ soit le plus souvent la vue. C'est pourquoi Lorca rappelle que, selon lui, la metáfora está siempre regida por la vista ${ }^{33}$. D'où la nécessité, face à une métaphore lorquienne, de prendre en compte l'image visuelle proposée par le référent (forme, aspect, dimension, couleur...) car très souvent l'intersection sémique entre comparé et comparant se fonde, en effet, sur un détail visuel sélectionné et amplifié.

Lautre aspect propre à l'image gongorine et par conséquent lorquienne, c'est que celle-ci doit être le résultat d'une " lucide et froide chasse nocturne ", car contrairement aux surréalistes et même à ce qu'il pourra affirmer, luimême, dans des conférences postérieures, il ne croit pas que los estados de fiebre favorisent la création. À partir de la longue allégorie de la cacería nocturna, Lorca explique, non sans quelques touches humoristiques, comment le poète doit rester maître de lui-même, "serein ", conscient et lucide comme Ulysse face au chant des Sirènes pour ne pas se laisser abuser par ces "mirages " que sont les "fausses métaphores", ces mil fealdades disfrazadas de belleza. Comme Góngora, Lorca aspire à être un chasseur de métaphores qui caza la que casi nadie ve, porque (los demás) la encuentran sin relaciones ${ }^{34}$; comme chez Rimbaud, le poète devient un "voyant".

Pour Lorca, l'image poétique n'est ni une imitation ni une reproduction mais une authentique création qui réunit surprise et familiarité, faisant fusionner l'inconnu et le connu, le distant et le proche, ce qui n'est pas sans nous rappeler le concept de "stupéfiant-image " utilisé par Aragon pour parler des images surréalistes et bien que, contrairement à ces dernières, elles soient le résultat d'une lucidité extrême. Lorca, incapable de dissocier le métapoétique et le poétique, décrit de façon métaphorique l'espace propre à la métaphore : para que una metáfora tenga vida necesita dos condiciones esenciales : forma y radio de acción. Su núcleo central y una redonda perspectiva en torno de él. El núcleo se abre como una flor que nos sorprende por lo

32. Ibid., p. 229.

33. Ibid., p. 230.

34. Ibid., p. 236. 
desconocida, pero en el radio de luz que la rodea hallamos el nombre de la flor y conocemos su perfume ${ }^{35}$. Pour définir la métaphore, Lorca a recours à une double métaphore synesthésique, celle d'une sphère avec son rayon de lumière et celle d'une fleur avec son parfum, lequel contrairement à la fleur insolite doit nous paraître familier.

Puis Lorca décrit le processus de création métaphorique en déclarant que la metáfora une dos mundos antagónicos por medio de un salto ecuestre de la imaginación $^{36}$. Cela suppose qu'il existe deux réalités, celle de la métaphore et celle de son objet, ou pour l'exprimer d'une manière plus rhétorique et afin de ne pas confondre les signes avec ce qu'ils désignent, le comparé et le comparant. Si bien que l'art inventif du poète est d'effectuer un "saut équestre" entre ces deux réalités qui peuvent être très distantes, voire opposées. Cette définition lorquienne n'est pas sans rappeler une citation de Pierre Reverdy dans Le gant de crin, - que récupérera plus tard André Breton en la modifiant -, selon laquelle : «Plus les rapports de deux réalités rapprochées seront lointains et justes et plus l'image sera forte, plus elle aura de puissance émotive et de réalité poétique " ${ }^{37}$.

Nous voyons bien que la métaphore ou l'image poétique - sans considération particulière de tropes - est un espace où se fondent des réalités contraires et que, seul, le poète est capable de créer des unités sémantiques originales, susceptibles de réconcilier des différences de taille, de forme, de couleur, de matière... et que la qualité de ces images dépend justement de la longueur du saut effectuée par l'imagination pour rapprocher ces réalités contraires. Pour Lorca, il existe une harmonie, une relation secrète entre les deux réalités, susceptible de révéler, pour qui comme Góngora la découvre, l'essence des choses.

Lorca nous montre également comment la métaphore embrasse tout et qu'il ne peut par conséquent y avoir de thème petit ou grand. C'est ainsi qu'il nous explique que pour Góngora una manzana es tan intensa como el mar, $y$ una abeja, tan sorprendente como un bosque ${ }^{38}$ et que le thème lui-même importe peu. Tout compte fait, le contenu est secondaire, car comme nous l'explique Lorca, usant de métaphores que n'auraient pas renié des poètes avant-gardistes : la grandeza de una poesía no depende de la magnitud del tema, ni de sus proporciones ni sentimientos. Se puede hacer un poema épico de la lucha que sostienen los leucocitos en el ramaje aprisionado de las venas ${ }^{39}$. Ce

35. Ibid., p. 230.

36. Ibid.

37. P. Reverdy, Le gant de crin, Paris, Librairie Plon, 1927, p. 32.

38. Op. cit., p. 233.

39. Ibid. 
que Lorca perçoit ici, c'est l'unité de l'univers où aucun élément ne semble vraiment isolé, car il est toujours en rapport avec ce qui l'entoure et vit les rythmes essentiels du cosmos.

Nous voyons donc quelles sont les leçons gongorines qu'a su exploiter Lorca. La première est sans nul doute la force d'une métaphore polysensorielle, capable de créer de la beauté et de conférer un sentiment d'éternité au poème. La seconde est une constante lucidité, sin apasionamiento peligroso, du poète à l'égard de son propre langage, toujours conscient de la calidad y sonoridad de la palabra. Une lucidité que conservera Lorca, même quand son écriture se rapprochera du surréalisme et lorsque des conférences comme Imaginación, inspiración, evasión ou Juego y teoría del duende accorderont plus d'importance aux forces instinctivistes, car jamais son écriture ne cessera d'aspirer à un certain contrôle, voire une "logique ». Mais il existe une troisième et double leçon que Lorca tire d'un Góngora aussi animiste que lui, c'est la création d'una letra que vivifique el espiritu et la langue, consciente de la nécessité d'inventer une nouvelle mythologie, c'est-à-dire un nouvel espace imaginaire.

Les images et/ou métaphores de Poema del Cante Jondo - bien que la rédaction du recueil soit antérieure à la conférence gongorine - sont une parfaite illustration de ce que nous venons de voir. En ce qui concerne la métaphore proprement dite, il convient, pour apprécier la justesse du "saut équestre de l'imagination", de déterminer à chaque fois le comparé, le comparant et l'intersection sémique qui les relie. En fonction de la syntaxe des phrases où sont employées les métaphores, on peut considérer qu'il existe trois grands types de métaphores : la métaphore nominale, la métaphore verbale et la métaphore adjectivale. Mais au sein de la métaphore nominale, il convient d'en distinguer plusieurs sous-groupes : la métaphore par apposition, la métaphore attributive - pratiquement inexistante dans ce recueil - et la métaphore du génitif, auxquelles j'ajouterai, bien évidemment, la métaphore in absentia et la comparaison.

- La métaphore par apposition: Cette catégorie est extrêmement fréquente dans ce livre. Elle consiste à apposer deux termes qui sémantiquement vont s'identifier l'un à l'autre, mais où souvent, l'un ou l'autre des termes de la métaphore peut être étendu à un groupe de mots. Nous trouvons cette métaphore par apposition pour conclure le poème « La guitarra » (p. 147) :

\author{
¡Oh guitarra! \\ Corazón malherido \\ por cinco espadas.
}


Le comparé est le terme guitarra et le comparant l'expression corazón malherido. L'intersection sémique est évidente pour qui a lu le poème qui commence avec : Empieza el llantol de la guitarra. Le sème commun est /llanto/ puisque dès le début la guitare est personnifiée et dotée d'un sentiment douloureux qui nous renvoie au cœur, considéré d'un point de vue figuré comme le lieu des émotions. La guitare «pleure " comme pleure un « cœur grièvement blessé ». En plus le comparant " corazón » fonctionne comme syllepse, c'est-à-dire à la fois au sens figuré - que nous avons déjà signalé - et au sens propre d'organe vital qui bat, qui marque un rythme comme peut le faire un instrument de musique. Quant au dernier vers qui désigne l'origine de la douleur (malheridol por cinco espadas), il contient une nouvelle métaphore, mais à présent in absentia, car seul apparaît de façon explicite le comparant cinco espadas alors que ne sont pas mentionnés les " cinq doigts " qui correspondent au comparé. Ici c'est le chiffre "cinq" qui indique qu'il s'agit des doigts de la main droite qui pincent les six cordes de la guitare. Le chiffre "cinq" fonctionne comme une correction métaphorique, c'est-à-dire comme une caractéristique du comparé ici éludé, appliquée au comparant. La correction métaphorique s'emploie souvent dans les métaphores in absentia pour faciliter leur compréhension. Ces " doigts » se transforment en " épées » car en pinçant violemment les cordes ou en y plaquant brusquement des accords, ils font que celles-ci semblent frappées, comme dans le rasgueo, mouvement violent, propre à la musique jonda, qui provoque un son pouvant s'identifier à une plainte ou à un llanto provenant de ce cour-guitare. Les termes malherido et espadas connotent métonymiquement la douleur inhérente au llanto et, en étant mis en rapport avec le mot corazón, ils nous rappellent l'image très andalouse d'un cœur transpercé par des épées ou des couteaux comme dans les représentations de la Virgen de los siete dolores, image d'un cœur qui ne cesse de saigner comme ne cesse de se faire entendre le llanto de la guitare.

Dans Poema del Cante Jondo, les métaphores par apposition ne se manifestent généralement pas au sein d'une même phrase. Parfois, seul un point sépare le comparé du comparant. C'est le cas de Crótalo./ Escarabajo sonoro ("Crótalo", p. 201). Dans cette métaphore le comparé crótalo, synonyme de "castagnettes" a pour comparant escarabajo sonoro. Limage repose sur une ressemblance visuelle entre comparé et comparant dont les sèmes communs pourraient être/forme bombée/ et /couleur sombre et brillante/. Mais le choix de ce signifiant proparoxyton crótalo montre que la métaphore est également motivée par l'aspect phonique puisque comparant et comparé partagent les sonorités des occlusives $(\mathrm{K} / \mathrm{T} / \mathrm{K} / \mathrm{B})$, de la liquide vibrante /R/ et des assonances en $/ \mathrm{A} /$ et $/ \mathrm{O} /$. Comme il s'agit d'un instrument 
de musique, ces phénomènes phoniques sont tout à fait appropriés sans parler du bourdonnement du scarabée lorqu'il se met à voler traçant des circonvolutions dans l'air comme le font les castagnettes d'une bailaora.

La plupart du temps, on trouve même, dans ce livre, un blanc entre le comparé et le comparant. C'est ce que nous proposent les poèmes "Chumbera» (p. 202) et "Pita» (p. 203) dont les titres correspondent chacun à un comparé tandis que chaque vers initial, Laoconte salvaje - référence à un ensemble sculptural connu - et Pulpo petrificado, fonctionne comme comparant. On peut observer que, dans la métaphore de la pita, l'identité fondée sur l'analogie entre les feuilles de la plante et les tentacules de l'animal est renforcée par une identité phonique grâce à l'allitération des occlusives et en particulier du /P/: "PiTal PulPo PeTrifiKaDo", alors qu'ici, contrairement à "Crótalo", le référent du comparé ne l'imposait absolument pas. Dans le poème "Chumbera ", le $4^{\mathrm{e}}$ vers, Múltiple pelotari, clairement détaché, propose un autre comparant grâce à la ressemblance visuelle et stylisée entre la forme du figuier de barbarie avec ses tiges ou branches semblables à des bras "multiples " et le joueur de pelote basque. Ressemblance accrue du fait que les tiges de ce cactus soient en "raquettes " comme la pala du pelotari et que ses fruits puissent rappeler la balle que celui-ci frappe.

Mais la métaphore par apposition ou juxtaposition peut se présenter de façon encore plus subtile. Tel est le cas dans «Amparo» (p. 185) où le mécanisme métaphorique peut passer inaperçu, et se trouve presque dissimulé typographiquement non seulement par un blanc qui sépare comparé et comparant - chacun se composant de divers éléments - mais par l'emploi des parenthèses. En réalité, il convient de remarquer que le comparé correspond au tercet qui ouvre le poème :

$$
\begin{aligned}
& \text { Amparo, } \\
& \text { iqué sola estás en tu casa } \\
& \text { vestida de blanco! }
\end{aligned}
$$

et le comparant au distique qui suit entre parenthèses :

$$
\begin{aligned}
& \text { (Ecuador entre el jazmin } \\
& \text { y el nardo.) }
\end{aligned}
$$

Les parenthèses sont un procédé, fréquemment utilisé par le poètedramaturge, qui fonctionne comme une indication scénique au sein du poème. Il n'est pas impossible que ce soit le cas, mais, ici, elles servent avant tout à introduire une explication-définition, sorte de commentaire poétique à ce que représente le personnage d'Amparo, défini grâce à la 
métaphore. L'intersection scénique se manifeste principalement à travers le sème /blancheur/, commun à la robe d'Amparo et aux fleurs citées ici - el jazmín y el nardo -, mais par connotation sont aussi présents d'autres sèmes : /beauté/, /fragilité/, /pureté/, /virginité/ et même /sensualité/ grâce au parfum de ces fleurs réputées pour leur fragrance enivrante et grâce au sème /chaleur/ suggéré par le mot Ecuador et par les zones géographiques des dites fleurs. La métaphore suggère qu'Amparo est comme une fleur au milieu d'autres fleurs avec toutes les connotations subséquentes. Quant au mot Ecuador - terme géographique qui fait allusion au centre, à la ligne ou frontière qui divise deux hémisphères, ici les deux types de fleurs -, il peut connoter une idée de symétrie ou d'harmonie. Enfin, il ne faut pas oublier, pour en revenir aux parenthèses, qu'elles peuvent symboliser dans cet exemple précis l'enfermement auquel est condamnée Amparo, personnage qui semble annoncer celui de la monja gitana.

Pour conclure sur la métaphore par apposition dans ce livre, examinons le très bref poème "Cruz " (p. 204) :

\section{La cruz. \\ (Punto final \\ del camino.) \\ Se mira en la acequia. \\ (Puntos suspensivos.)}

Le tercet inclut une première métaphore où le premier vers, $L a$ cruz, est le comparé et les vers 2 et 3 , entre parenthèses, sont le comparant. L'intersection sémique explicite est / finall. En effet, la cruz nous renvoie à la fin de la vie du Christ et de celle de l'homme - pensons à la croix qui surmonte la sépulture chrétienne - et le punto à la fin d'un texte. L'idée de fin fait subtilement écho à la place de ce texte au sein du livre et permet de mettre "un point final " à ce dernier, avant les diálogos. Ensuite, dans le distique le substantif cruz, parce qu'il devient sujet du verbe se mira, disparaît, escamoté par son reflet dans l'eau du canal. De sorte que le reflet de la croix est à présent le comparé d'un nouveau comparant qui correspond au dernier vers, lui aussi entre parenthèses: (Puntos suspensivos). Tout laisse supposer que l'image se fonde sur le reflet de la croix qui, à cause de la diffraction des ondes, doit apparaître comme fragmenté, semblable à une suite de "points de suspension ". Mais le plus intéressant ici, c'est sans doute la réflexion métapoétique apportée par le poème qui grâce au comparant puntos suspensivos se conclut sur une image propre au travail de l'écriture et qui suppose non une fermeture définitive mais une possible ouverture, celle de ce poème et du livre où il s'insère, 
comme si la référence imagée à la ponctuation pouvait symboliser une éventuelle résurrection. Une résurrection qui ne serait plus celle du Christ mais du texte poétique lui-même. Nous voyons ainsi que la métaphore lorquienne n'est pas ornementale mais qu'elle joue souvent un rôle essentiel pour l'existence même du poème et sa signification.

- La métaphore du génitif: Si la métaphore attributive, fondée sur le verbe " être " suivi d'un prédicat, est relativement courante dans Libro de poemas ainsi que dans Poeta en Nueva York et Diván del Tamarit, elle est pratiquement inexistante dans ce recueil et dans Romancero gitano, comme si Lorca y préférait à chaque fois la définition par apposition. En revanche, la métaphore du génitif, qui a recours à la préposition de, est un type de métaphore qui abonde dans Poema del Cante Jondo comme dans d'autres recueils lorquiens. Le poème «El grito » (p. 148) s'ouvre avec une métaphore du génitif, La elipse de un grito, qui sert à visualiser la trajectoire prolongée et parabolique du cri. Or, il est très intéressant de voir comment le sème /courbe/ de cette image permet au poème de s'écrire avec d'autres métaphores de ce cri, dotées, elles aussi, du même sème. C'est ainsi que nous découvrons dans le deuxième tercet une des très rares métaphores attributives : será un arco iris negro/ sobre la noche azul puis dans le troisième une comparaison qui renvoie également au sème /courbe/ : Como un arco de violal el grito ha hecho vibrarl las cuerdas del viento. Dans le poème "Sevilla " (p. 167), nous trouvons, pour évoquer une vue panoramique, le même type d'image avec une métaphore filée ou métaphore à quatre termes : bajo el arco del cielo,/ sobre su llano limpio,/ dispara la constantel saeta de su río. Les comparés sont cielo et río et leurs comparants, arco et saeta au sens de flèche, bien que ce signifiant prépare la venue d'une autre saeta. Dans le Romancero Gitano, recueil proche de ce livre, la métaphore du génitif est omniprésente comme dans el tambor del llano ou dans la rosa azul de tu vientre. Dans les exemples empruntés à ces deux livres le génitif correspond à chaque fois au comparé. Mais cela peut s’inverser faisant alors du génitif le comparant. C'est le cas dans les didascalies finales de «Escena del Teniente Coronel de la Guardia civil » (p. 209) lorsqu'après s'être s'effondré mortellement blessé par la fantaisie créatrice du gitan, le militaire rend l'âme : (El alma de tabaco y café con leche del Teniente Coronel de la Guardia civil sale por la ventana.). Dans cette métaphore le comparé est el alma tandis que le génitif de tabaco y café con leche correspond au comparant. Cette métaphore visuelle suggère la couleur sombre et trouble de l'âme du colonel, en ayant même recours à d'autres sens tel le goût et l'odorat, afin de montrer son âme comme quelque chose de volatil, telle une fumée ou une vapeur qui s'échapperait par la fenêtre. Lorca apparaît bien comme ce profesor en los cinco sentidos corporales 
qu'il recommande à tout poète d'être dans sa conférence gongorine. Dans le Romance de la Guardia Civil, on trouve aussi plusieurs métaphores où le génitif devient le comparant. Ainsi lisons-nous El alma de charol, métaphore qui permet de caractériser une fois de plus l'âme sombre et insensible des gardes civils grâce à ce matériau noir et imperméable qu'est le charol avec lequel sont fabriqués leurs tricornes. Puis, plus bas, nous avons d'autres métaphores pour désigner les agissements répressifs de ces personnages, où les génitifs sont des comparants qui permettent de caractériser de façon concrète et sensorielle des comparés abstraits : ordenan/ silencios de goma oscural y miedos de fina arena.

- La métaphore in absentia : En admirateur de Góngora, Lorca utilise souvent cette métaphore, où seul apparaît de façon explicite le comparant tandis que le comparé est omis quoique implicite. Nous la trouvons dans le poème "Procesión" ( $\mathrm{p} 169)$ : Por la calleja vienen/ extraños unicornios; vu le contexte indiqué par le titre, il est facile de comprendre que cette métaphore in absentia se réfere aux pénitents qui participent aux processions de la Semaine Sainte. Cette image humoristique se fonde sur les cagoules coniques des pénitents qui apparaissent ainsi comme des êtres étranges munis d'une longue corne. Dans le poème "Camino " (p. 176) qui évoque le lieu où se rendent les cien jinetes, nous avons également une métaphore in absentia, que nous avions déjà relevée : Esos caballos soñolientos/ los llevarán, al laberinto de las cruces, cette image métaphorise un lieu précis : le cimetière où sera enterrée la Petenera et où iront échouer, eux aussi, les cavaliers. La métaphore in absentia se manifeste aussi dans "Danza " (p. 178) avec les Seis gitanas,/ vestidas de blanco:/ (que) bailan et qui évoquent les cordes de la guitare qui vibrent comme si elles dansaient tandis que la musique se fait entendre. Très souvent ces métaphores in absentia sont assorties d'une correction métaphorique, un élément appartenant au comparé omis quoique implicite, pour faciliter la compréhension, il s'agit ici du chiffre seis qui renvoie au nombre de cordes que possède la guitare. Le même procédé, avec le même comparé se référant aux cordes mais métaphorisé par d'autres comparants, est employé dans les poèmes "Adivinanza de la guitarra " (p. 199) avec les seis doncellas (que) bailan en la redonda encrucijada et "Barrio de Córdoba " (p. 197) avec Seis ruiseñores la lloran/ en la reja. Dans ce "Tópico nocturno " - c'est le sous-titre de ce dernier poème - qui évoque la veillée funèbre d'une "enfant morte ", les cordes par le chant douloureux qu'elles expriment s'identifient à des « rossignols». Mais l'image n'est qu'un topique réactivé, car il y a subversion de la fonction habituelle et joyeuse de la sérénade chantée près de la reja. Par ailleurs cette image andalouse de la fenêtre et de sa reja est assimilée ici à celle de la rosace de la guitare 
dont l'entrée semble protégée par ces barreaux que sont les cordes, lesquelles, par leur chant, se métamorphosent en "rossignols ", symboles de l'amour malheureux pour pleurer mélodiquement la mort de l'enfant.

- La comparaison : contrairement à la métaphore (similitudo brevior), la comparaison est une image où apparaissent de façon explicite trois éléments : le comparé, le comparant et l'introducteur syntaxique - en principe dans ce livre como - qui différencie cette figure d'une vraie métaphore. Ce trope est très souvent employé dans l'œuvre poétique lorquienne, et tout particulièrement dans Poema del Cante Jondo. Il est présent dans "Paisaje» (p. 144) : El campol de olivos/ se abre y se cierral como un abanico, image dynamique qui suppose différentes rangées d'oliviers subissant les assauts successifs de fortes rafales de vent. La comparaison se manifeste doublement dans le poème « La guitarra " (p. 146), faisant de l'introducteur syntaxique como une anaphore qui prolonge dans ces vers l'assonance sombre du /o/ : Llora monótonal como llora el agual como llora el viento. Dans « Puñal» (p. 156) nous trouvons une double comparaison de l'arme : El puñall entra en el corazón/ como la reja del arado/ en el yermo et El puñal,/ como un rayo de sol,/ incendia las terribles/ hondonadas. La comparaison en ayant recours à l'hyperbole et à des images empruntées au paysage andalou intensifie le pouvoir de l'arme.

Contrairement à la métaphore où les deux éléments ou mondes fusionnent pour créer une nouvelle réalité, dans la comparaison - cette figure qui a des propriétés de "suspension " comme dirait André Breton ${ }^{40}$ - les deux éléments subsistent. Si la métaphore marque par sa fulgurance, la comparaison, elle, fait en sorte que les deux termes se tolèrent avec un maximum de force. C'est un peu comme si la comparaison avait le pouvoir de sauvegarder les deux mondes, à la fois le regard et ce sur quoi est porté ce regard. Mais cela n'empêche pas l'introducteur syntaxique d'intensifier les propriétés de ces deux mondes.

- La métaphore verbale: Cette métaphore peut ou non être accompagnée d'un complément. Dans ces métaphores, le verbe, en désignant une action ou un état, possède un sujet qui est agent ou patient. Cela contribue à faire de la plupart de ces images des métaphores antropomorphiques, des personnifications ou des prosopopées. Cette métaphore est déjà présente dans Poema del Cante Jondo mais elle reste assez discrète. On la trouve dans "Noche " (p. 166) pour suggérer la lueur tremblante des fenêtres éclairées

40. André Breton écrit : "Il ne saurait être fait grand état de la distinction purement formelle qui a pu être établie entre la métaphore et la comparaison. Il reste que l'une et l'autre constituent le véhicule interchangeable de la pensée analogique et que si la première offre des ressources de fulgurance, la seconde [...] présente de considérables avantages de suspension. ", in Signe ascendant, Paris, Poésie/ Gallimard, 1968, p. 10. 
dans la nuit : Ventanitas de orol tiemblan ou dans "Malagueña " (p. 196) pour personnifier la mort : La muertel entra y salel de la taberna, bien qu'on puisse expliciter l'image par le titre musical du poème qui laisse supposer que c'est une musique avec son chant de mort qui entre et s'échappe du cabaret. Ce type de métaphore tend très souvent vers l'allégorisation et certains poèmes du livre constituent en effet de petites et délicates allégories. Mais ces métaphores verbales donnant lieu à des prosopopées vont surtout être présentes dans Romancero gitano pour engendrer, dans des poèmes narratifs et dramatiques, de véritables personnages, qu'il s'agisse de la nuit ( $L a$ noche llama temblando/ al cristal de los balcones, in " Muerto de amor ", p. 269), de la lune (Por el cielo val la luna con un niño de la mano, in "Romance de la luna, luna », p. 226) ou du vent : El viento, furioso, muerde ("Preciosa y el aire " p. 229) : ces poèmes montrent que la métaphore anthropomorphique, presque toujours verbale, constitue une véritable métamorphose.

- La métaphore adjectivale: On peut observer que dans ce livre Lorca n'utilise jamais ce type de métaphore, où l'adjectif pourrait former avec le substantif une sorte d'oxymore. Ailleurs, en particulier dans Romancero gitano, il ne l'utilise à vrai dire, que très rarement, comme dans la corta brisa ecuestre de "Prendimiento de Antońito el Camborio... ». Car Lorca semble presque toujours lui préférer la métaphore du génitif où celui-ci renvoie à un mot concret permettant de matérialiser l'abstrait.

Chez Lorca, la métaphore constitue le moteur même du poème, participant ainsi à son mouvement et à son élaboration. Très souvent elle est, comme nous avons pu l'observer, associée à d'autres figures ou part de cellesci. D'où l'importance lorsque l'on considère l'image poétique lorquienne d'autres tropes comme la métonymie, la synesthésie ou l'hypallage. Le poème "Amparo " nous offre un exemple d'une image où se combinent synesthésie et hypallage. Le locuteur interpellant Amparo lui dit : Oyes.......... el débil trino amarillo/ del canario. On peut observer, en effet, que la synesthésie, qui vient confondre ici une sensation auditive trino avec une sensation visuelle amarillo, se fonde, en fait, sur une hypallage puisque le jaune qui caractérise la couleur du plumage du canari passe par contiguïté ou métonymie à son chant. Cette synesthésie permet d'exprimer une sensation de beauté tout en insistant sur une sensorialité exacerbée. L'image joue toujours un rôle précis et fonctionnel au sein du poème. C'est par ses sens qu'Amparo, comme la monja gitana du Romancero gitano, peut se soustraire à son enfermement et ce n'est donc pas un hasard si l'oiseau évoqué ici est le canari, un oiseau qui est une métonymie de la cage. De sorte que celui-ci symbolise, lui aussi, l'enfermement et le qualificatif débil, qui suggère un état faible ou maladif, insiste donc à la fois sur la fragilité ou la délicatesse du chant et sur une sorte 
de maladie, celle de l'oiseau mis en cage. Enfin, n'oublions pas que le « jaune » est traditionnellement la couleur qui symbolise l'amour malheureux.

Nous voyons donc que l'image poétique lorquienne est le fondement même du poème et du recueil qui l'accueille. Cette image polysensorielle et animiste souvent associée au symbole, vitalise la matière, donne une densité concrète et animée à ce qui est abstrait, n’hésite pas parfois, par le biais de l'hyperbole, à devenir humoristique et s'ouvre à plusieurs tropes à la fois. Il faut toujours - et c'est encore plus vrai pour le Romancero gitano - mettre en rapport la métaphore avec la structure même du poème car certaines images obsédantes peuvent en constituer l'histoire interne. Enfin, n'oublions pas que l'importance, dans la poésie lorquienne, de la prosopopée tend souvent vers l'allégorisation comme dans Poema del Cante Jondo ou vers une narration qui devient mythe comme c'est le cas dans le Romancero gitano.

\section{LECTURE LORQUIENNE DE LA COPLA}

(SIGUIRIYA, SOLEÁ, SAETA, PETENERA...)

Comme l'indique son titre Poema del Cante Jondo, ce livre - bien qu'il soit l'œuvre d'un poète savant - revendique une origine populaire et musicale précise, laquelle est confirmée ensuite par les titres des quatre sections majeures qui servent de fondations au livre, puisque chaque titre se réfère à une forme populaire spécifique : la siguiriya, la soleá, la saeta et la petenera. Ces formes poétiques et musicales désignent des coplas chantées par les cantaores et dont les paroles sont en principe anonymes. Mais ce qui attire d'emblée notre attention, c'est que la plupart du temps les poèmes lorquiens ne semblent pas coïncider exactement avec ces formes traditionnelles qui donnent leur nom à chacune des quatre sections principales du livre.

$\mathrm{Si}$ nous nous référons, par exemple, à la siguiriya, à aucun moment n'apparaît, dans la section intitulée par Lorca Poema de la siguiriya gitana, la forme poétique que suit habituellement la copla qui correspond à la siguiriya ou seguidilla gitana.

En réalité l'appellation siguiriya gitana est pléonastique car le qualificatif ne se justifie vraiment que lorsqu'il est apposé au terme seguidilla pour différencier la séguedille gitane - et dans ce cas le terme siguiriya, reflet de la prononciation populaire, suffirait - de celle qui ne l'est pas. La seguidilla non gitane se présente, elle, comme une cuarteta où alternent des pentasyllabes et des heptasyllabes aux rimes assonancées à la fin des vers pairs, les impairs étant blancs ou plus exactement sueltos. Cette strophe de la poésie populaire correspond, lorsqu'il s'agit d'une seguidilla simple au schéma métrique 
suivant: $7 \mathrm{x}-5 \mathrm{a}-7 \mathrm{x}-5 \mathrm{a}$. Mais dans certains cas, il est possible d'ajouter à cette strophe pour obtenir une seguidilla compuesta un bordón facultatif avec une nouvelle rime assonante : $5 \mathrm{~b}-7 \mathrm{x}-5 \mathrm{~b}$. Il est arrivé à Antonio Machado d'employer ces formes :
Por las tierras de Soria va mi pastor. ¡Si fuera una encina sobre un alcor!

$$
\begin{aligned}
& \text { + bordón : Para la siesta } \\
& \text { si yo fuera una encina } \\
& \text { sombra le diera. }
\end{aligned}
$$

On peut trouver parfois un hexasyllabe à la place du premier heptasyllabe.

Quant à la seguidilla gitana ou siguiriya, cette copla employée dans les chansons andalouses jondas, elle correspond en principe à une cuarteta assonancée aux vers pairs, dans laquelle le premier, le second et le quatrième vers sont des hexasyllabes, et le troisième, le plus souvent, un hendécasyllabe et occasionnellement un décasyllabe, voire un dodécasyllabe. Son schéma métrique est donc généralement le suivant : 6x-6a-11x-6a. Lauteur de la siguiriya est le plus souvent anonyme, bien que certains poètes savants comme Manuel Machado - qui écrivit un Cante Hondo - aient pu y recourir. En revanche, nous ignorons l'identité, par exemple, de celui qui écrivit les siguiriyas suivantes :

\author{
Cuando viene el día \\ mis penas se agrandan \\ sólo las sombras de noche oscura \\ consuelan mi alma. \\ Cuando yo me muera \\ mira que te encargo \\ que con la cinta de tu pelo negro \\ me amarren las manos.
}

Il convient de remarquer qu'aucune soi-disant siguiriya de la section intitulée Poema de la siguiriya gitana n'adopte ce type très précis de strophe. On peut observer, par ailleurs, que lorsque Lorca fait l'éloge, dans sa conférence sur El Cante Jondo, de ce perfecto poema de las lágrimas qu'est, selon lui, la siguiriya gitana, le poète, peu respectueux du légitime canon métrique, nous donne des exemples de cuartetas octosyllabiques assonancées qui correspondent à ce qui dans la poésie populaire se définit comme coplas :

De noche me sargo ar patio y me jarto de llorá, en ver que te quiero tanto y tú no me quieres ná. 
En effet, la copla est généralement un quatrain assonancé aux vers pairs, les vers impairs étant non rimés, c'est-à-dire sueltos. Un quatrain presque toujours octosyllabique bien qu'il admette, occasionnellement, des vers plus courts, en particulier des hexasyllabes. Par ailleurs, il convient de remarquer que la copla, très répandue à travers toute l'Espagne, peut porter, suivant les traditions régionales ou folkloriques, des noms divers comme jota (aragonesa), ronda (manchega), granadina et, plus intéressant pour nous, ceux de saeta, petenera ou malagueña. Rappelons aussi, au passage, que la copla - qui au moyen-âge désignait simplement une strophe - peut également constituer en elle-même un très bref poème.

Il convient d'observer, cependant, que si la saeta est une forme poétique chantée qui correspond à la copla, elle se définit aussi en fonction de son thème et des circonstances qui accompagnent son chant. De sorte que la saeta, forme typiquement sévillane dont le thème est presque exclusivement religieux, est une sorte d'oraison jaculatoire qui est chantée à l'église et surtout dans les rues lors des processions de la Semaine Sainte. Lancée ou décochée à voix haute comme une flèche, d'où son nom qui a le même étymon que sagette, ses paroles rappellent généralement la passion et la crucifixion du Christ. Son chant doit s'élever dans l'espace et pénétrer le cour de ceux qui possèdent une vive foi chrétienne. Or, si Lorca respecte en partie le contexte de ce chant - bien que ce soit en le subvertissant, comme nous l'avons vu, d'un point de vue profane -, il se permet aussi certaines libertés par rapport au canon de la copla comme cuarteta assonancée et nous pourrions en dire tout autant des poèmes qui apparaissent dans la section intitulée Gráfico de la petenera.

La petenera est traditionnellement, elle aussi, un quatrain octosyllabique assonancé aux vers pairs, qui se chantait à Paterna, village de la province d'Almería. Sa mélodie à l'aspect mélancolique et languissant est généralement mise en évidence par la répétition d'un ou plusieurs vers, principalement ceux qui ouvrent le chant, afin de souligner ou de rappeler l'air principal. Or, il convient, en effet, d'observer que, mis à part le poème "Las seis cuerdas ", les sept autres poèmes restants de cette section obéissent à cette caractéristique avec des vers qui fonctionnent comme des refrains isolés ("Muerte de la Petenera ") ou comme la reprise du même vers au début de chaque cuarteta ( Danza ) ou avec des vers identiques pour ouvrir et refermer la composition ("Falseta », "De profundis» et, d'une certaine façon, "Clamor»).

Quant à la soleá - andalousisme pour soledad, qui devient soleares au pluriel -, il s'agit d'une copla très particulière de la poésie populaire andalouse, sorte de tercet qui ne compte que trois vers octosyllabiques avec des rimes 
assonantes aux vers impairs selon le schéma métrique suivant : 8a-8x-8a. La soleá est chantée avec un air mélancolique et sur une mesure à $3 / 8$, ce qui produit un rythme vif, approprié pour la danse. Généralement les soleares sont anonymes comme celles-ci :

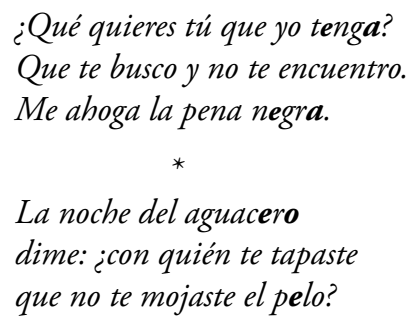

Si nous examinons de près la section Poema de la soleá, nous pouvons observer que le poème intitulé précisément " La soleá » (p. 160) - qui évoque la forme poétique personnifiée sous les traits d'une femme en deuil - est une authentique soleá, ou plus exactement la réunion de trois soleares assonancées en /é-o/, avec la répétition du vers initial transformé en refrain :

\section{Vestida con mantos negros piensa que el mundo es chiquito y el corazón es inmenso.}

Il est également important de remarquer que le poème précédent, "Sorpresa " (p. 159), où est racontée la cause du deuil sous la forme de l'assassinat d'un inconnu poignardé en pleine rue, était déjà en partie - bien que la disposition typographique ne l'indique pas - une soleá ou un ensemble de trois soleares assonancées en /á-e/, dont la première est :

\section{Muerto se quedó en la calle con un puñal en el pecho. No lo conocia nadie.}

Mais, avant de passer aux deux autres soleares, vient s'intercaler entre les vers 4 et 7 une cuarteta assonancée qui correspond à l'expression de stupeur de celui qui a découvert le corps :

$$
\begin{aligned}
& \text { ¿Cómo temblaba el farol! } \\
& \text { Madre. } \\
& \text { ¡Cómo temblaba el farolito } \\
& \text { de la calle! }
\end{aligned}
$$

Par ailleurs, on peut observer que si certains poèmes de cette section - la plupart d'entre eux - s'écartent délibérément du modèle canonique de la soleá, d'autres, qui typographiquement forment un seul bloc assonancé, 
jouent - si nous prêtons attention à la syntaxe et au sens des phrases - sur de discrètes et possibles cuartetas provenant de la copla et sur quelques autres inspirées, elles, de la soleá, situées soit à l'ouverture ou à la fin d'un poème (ex : Ni tú ni yo estamos/ en disposición/ de encontrarnos. in "Encuentro", $\mathrm{p} 162)$ soit en son milieu (ex: Os sienten todas las muchachas/ que lloran a la tiernal soleá enlutada, v 5-7, p. 163).

Mais ces soleares virtuelles et masquées sont réalisées, bien évidemment, avec une plus grande souplesse car elles pratiquent souvent la polymétrie. Le fait est que Lorca joue sur ses formes populaires transformées et revisitées en fonction de sa liberté créative.

C'est ainsi qu'il n'est pas impossible de voir au début et à la fin de "Amparo " (p. 185, le second poème de Dos muchachas, une utilisation personnelle de la soleá tandis que le centre du poème est occupé, lui, par la copla conçue comme cuarteta octosyllabique assonancée. De sorte que les apostrophes adressées par le locuteur à la solitaire et recluse Amparo s'énoncent toutes avec une soleá "libre ou libérée ", ce qui fait coïncider subtilement forme et signification :

\section{Amparo, \\ ¿Qué sola estás en tu casa vestida de blanco!}

Si bien que, mise à part la forme poétique de la soleá qui se manifeste dans certains poèmes de la section consacrée à cette strophe, la cuarteta précise de la siguiriya $(6 \mathrm{x}-6 \mathrm{a}-11 \mathrm{x}-6 \mathrm{x})$ ou la copla définie comme cuarteta octosyllabique et assonancée inhérente à la saeta et à la petenera ne sont respectées dans aucune des sections qui se réclament de ces trois formes poétiques.

Cependant, il est évident qu'il y a de constantes allusions à ces coplas et bien que le poème se présente souvent d'un seul bloc, il est facile de voir comment on pourrait, à plusieurs reprises, détacher des cuartetas assonancées rien qu'en créant certains espaces typographiques. Nous pouvons observer que le poème consacré à la siguiriya, intitulé "El paso de la siguiriya", choisit comme patron strophique la cuarteta octosyllabique et assonancée de la copla que nous pourrions attendre dans d'autres sections tandis que le poème antérieur, "El silencio" (p. 149), semble déjà annoncer la soleá puisqu'il semble formé par deux tercets assonancés aux vers impairs. Lorca part d'une inspiration populaire représentée par les diverses formes de copla mais en les transformant pour se les approprier.

C'est ainsi que, même dans un poème comme "Muerte de la Petenera " (p. 179-180), qui suit en grande partie la cuarteta octosyllabique assonancée de la copla, la dernière strophe a recours, après trois octosyllabes, à un 
verso quebrado trisyllabique qui convient parfaitement pour souligner mimétiquement la rupture du bordón de la guitare :

$\begin{array}{ll}\text { Largas sombras afiladas } & 8 x \\ \text { vienen del turbio horizonte, } & 8 a \\ \text { y el bordón de una guitarra } & 8 x \\ \text { se rompe. } & 3 a\end{array}$

Néanmoins, dans presque tous les poèmes nous pouvons relever une caractéristique propre à la poésie populaire qui est l'omniprésence de la rime assonante, située la plupart du temps aux vers pairs. Si nous mettons à part la dernière section où figurent deux dialogues très postérieurs aux textes précédents et accompagnés, en plus, de deux canciones assonancées, sur les 51 poèmes du livre, seuls six d'entre eux n'ont pas de rime assonante, lesquels se réduisent à deux si nous ne retenons que les quatre sections majeures qui ouvrent le recueil. Et ces deux poèmes dépourvus de rime ("El grito » p. 148, et " iAy!" p. 158) sont particulièrement symboliques, car ils sont tous deux consacrés au cri, à la manifestation de quelque chose d'immédiat et de spontané, comme antérieur à tout système. Les quatre poèmes restants non rimés appartiennent à la section très conceptiste Seis caprichos et sont "Conjuro", "Candil», "Chumbera " et "Pita », bien qu'il ne soit pas impossible de lire dans ce dernier des "rimes inversées " (é-a/á-e, ó-e/é-o).

Ces poèmes, tous inspirés de la copla, laquelle peut être à la fois une strophe et un poème, c'est-à-dire une micro et une macrostructure, suggèrent la volonté de la part de Lorca de tendre, dans ce recueil, vers un type de poème plutôt court, voire bref où vont dominer la condensation et la concentration. Cette volonté de concision qui obéit à un véritable travail d'épure, nous la découvrons aussi dans les poèmes qui ont recours au distique. Si le quatrain ou le tercet semblent fonctionner comme unité sémantique, il convient d'observer qu'apparaissent parfois de véritables distiques mis en évidence par des blancs typographiques qui suggèrent des silences ou des pauses. C'est le cas dans des poèmes comme "El grito ", "Y después ", " iAy! ", "Arqueros » et surtout "Cueva ». Cependant, on peut remarquer que ces distiques n'acquièrent presque toujours leur sens que par rapport à d'autres vers qui les suivent, distiques ou non. C'est le cas par exemple de «Cueva» (p. 161), poème où les distiques entre parenthèses fonctionnent comme indications scéniques pour créer l'ambiance ou clarifier ou gloser ce qui vient d'être évoqué dans le distique antérieur non parenthésé. C'est ainsi que dans ce poème les distiques entre parenthèses contiennent tous une référence au rouge, couleur de la passion : 


\author{
De la cueva salen \\ largos sollozos. \\ (Lo cárdeno \\ sobre lo rojo.)
}

En réalité, le livre ayant choisi, comme pour les romances, la rime assonante pour les vers pairs - et non la rime plate -, le distique sous-entend, ici, à la fois une séparation et un lien presque toujours confirmé sémantiquement qui suppose, lui, l'existence d'une possible cuarteta fragmentée pour insérer un silence ou un blanc qui iconiquement peut renvoyer à l'infini.

Si nous prêtons attention, à présent, à la longueur des poèmes de ce livre, nous pouvons remarquer que ceux-ci vont de 5 à 32 vers. Mais le plus long, celui de 32 vers, "Baladilla de los tres ríos" (p. 142), fondé sur la cuarteta octosyllabique assonancée assortie de quelques refrains, constitue une véritable exception et sa situation initiale comme prologue et prélude peut justifier son extension. Ensuite, le poème le plus long est "La guitarra " (p. 146) qui compte 27 vers tous très courts et dont l'extension est également exceptionnelle dans ce recueil où la longueur moyenne d'un poème n'atteint pas les 15 vers.

Sur les 51 poèmes du livre - sans considérer la quatrième section où deux canciones de 10 et 12 vers suivent les dialogues -20 poèmes ne dépassent pas les 13 vers et 11 les 10 vers. 6 poèmes comptent 10 vers, 2 en atteignent 9 et 2 autres encore simplement 6. Quant au poème conclusif "Cruz" (p. 204), il se limite à 5 vers très brefs. Or, si les poèmes tendent donc vers la réduction, ce phénomène est encore plus évident si nous nous intéressons à la métrique avec d'évidentes conséquences sur la syntaxe.

En effet, dans ce recueil s'impose un vers fluctuant - plus que libre -, très court qui va du dissyllabe, utilisé 30 fois, au décasyllabe qui n’est présent que 3 fois. Il ne nous semble pas inutile de recenser les occurrences de chaque mètre :

- dissyllabe $(30 \mathrm{x})$, trisyllabe $(49 \mathrm{x})$, tétrasyllabe $(104 \mathrm{x})$, pentasyllabe (99x), hexasyllabe (101 x), heptasyllabe (204x), octosyllabe (171 x), ennéasyllabe $(16 \mathrm{x})$, décasyllabe $(3 \mathrm{x})$.

Il ressort de ce recensement que le vers dominant est l'heptasyllabe et ensuite l'octosyllabe, employés respectivement dans la seguidilla et la copla. Mais le plus intéressant, c'est :

a) le désir de ne jamais employer l'hendécasyllabe - le vers savant par excellence - bien qu'il soit présent comme troisième vers de la siguiriya et

b) l'extrême abondance, au sein des vers d'arte menor, de mètres excessivement courts, et si quelques uns comme l'hexasyllabe ou le 
pentasyllabe s'emploient dans la siguiriya ou la seguidilla, ce n'est pas le cas d'autres (tétra-, tri- ou dissyllabes) très peu utilisés - excepté en de rares occasions chez les romantiques - et jamais dans de telles proportions.

Ces vers extrêmement courts supposent un important travail de condensation et de concentration, aussi bien horizontal que vertical, qui exige une rigoureuse ascèse langagière avec des phrases très brèves, souvent parataxiques et même parfois nominales.

Par ailleurs, ces vers très courts se prêtent, dans ce livre, à une polymétrie plus fluctuante que libre dans la mesure où presque toujours semblent dominer ou se détacher certaines séries de mètres. Car la polymétrie d'un vers à l'autre ne diverge le plus souvent que d'une seule syllabe. C'est pourquoi nous n'avons pas l'impression d'avoir affaire à un vrai vers libre à la longueur variable sans aucune structure métrique régulière. Un poème très polymétrique comme "La guitarra » (p. 146) où se mêlent tri-, tétra-, penta-, hexa-, hepta- et octosyllabes montre bien comment les mètres tendent à se répondre ou à se regrouper sans jamais provoquer d'écart important entre deux vers contigus.

Si certains vers brefs, aux rimes assonantes peuvent rappeler l'inspiration populaire du livre, le vers bref fluctuant peut, à notre avis, s'entendre comme une interprétation de ce que, dans sa conférence sur El Cante Jondo, Lorca appelle el enharmonismo, como medio modulante. Dans la musique ancienne l'enharmonie était un moyen modulant, un passage qui procédait par quarts de ton successifs. Or, el enharmonismo utilisé par les cantaores peut, selon Lorca, producir la impresión de una prosa cantada, destruyendo toda sensación de ritmo métrico, aunque en realidad son tercetos o cuartetos asonantados los textos de sus poemas ${ }^{41}$. C'est pour cette raison qu'ici, il nous semble que les légères variations entre un pentasyllabe et un tétrasyllabe ou entre ce dernier et un trisyllabe sont ce qui peut le mieux donner l'impression d'une "prose chantée ", non enfermée dans un rythme métrique trop prévisible, bien que demeure présente une certaine mesure plus subtile, juxtaposant des mètres aux variations discrètes.

En ce qui concerne le rythme du vers - bien que celui-ci soit, lui aussi, très variable -, il est fondamental d'observer que nombreux sont les poèmes où les vers sont dépourvus d'anacrouse. En effet, une très grande quantité de poèmes ont une majorité de vers accentués dès la première syllabe, ce qui leur donne un aspect emphatique et abrupt qui insiste sur l'émotion. C'est ainsi que la proportion des vers accentués sur la première syllabe par rapport à d'autres s'ouvrant sur une anacrouse s'élève, par exemple, à 10 sur 14 dans

41. "Arquitectura del Cante Jondo », op. cit., p. 220. 
"Paisaje " (p. 144), à 10 sur 16 dans « El paso de la siguiriya " (p. 150) ou à 10 sur 17 dans « Evocación» (p. 153).

Un poème apparemment long pour ce livre, comme "La guitarra " (p. 146), présenté typographiquement d'une seule pièce, qui semble néanmoins se structurer à partir de cuartetas - la plupart composées de deux phrases syntaxiquement indépendantes qui différencient la cause et l'effet -, et qui se conclut par un possible tercet, porteur de la métaphore définitoire de l'instrument, accumule sur un ensemble de 27 vers rien de moins que 11 vers accentués sur la première syllabe.

Toutes ces observations montrent clairement qu'il existe dans ce livre un très grand effort de concision qui semble s'inspirer de la force " condensatrice " de la copla, bien que typographiquement le poème ne se fragmente pas forcément en strophes. Quant aux poèmes qui n'excèdent pas les 10 vers ou à peine et qui constituent la plus grande partie du recueil, ils suggèrent, à notre avis, bien que cela ne soit pas signalé par la critique, une orientation vers la forme épigrammatique.

Dans l'antiquité une épigramme était, comme le rappelle Alain Montandon: "une inscription gravée sur un monument, plus particulièrement sur un tombeau ou un socle de statue, et destinée à donner sous une forme "lapidaire" un renseignement sur le personnage enseveli ou statufié $"{ }^{42}$. Cette relation entre la concision du style et le funéraire n'est pas dépourvue d'intérêt lorsque nous constatons que nombreux sont les poèmes de "Poema del Cante Jondo " qui entretiennent un rapport étroit avec la mort.

Montandon nous explique que "rapidement l'épigramme se libère de ses origines pour désigner tout poème concis et bref, la plupart du temps caractérisé par une ironie mordante ". Puis Montandon, après avoir passé en revue des auteurs anciens comme Catulle et Martial, rappelé la floraison de ce genre à l'époque baroque et défini son mécanisme en fonction du suspense et de la surprise, conclut en ces termes : "D’une manière très générale, la forme binaire de l'épigramme, sa concision et sa pointe finale, dépasse le cadre même du genre pour désigner une forme épigrammatique propre à de nombreuses poésies brèves " car comme il l'explique auparavant "l'épigramme peut également avoir quatre, six, huit vers ou parfois plus ${ }^{43}$.

Et si quelques poèmes baroques et conceptistes comme «Adivinanza de la guitarra » ou «Cruz » sont d'évidentes épigrammes, jusque dans l'emploi

42. Les formes brèves, Paris, Hachette, 1992, p. 15.

43. Ibid., p. 18. 
conceptiste plein d'agudeza de l'image, nous croyons que la plupart des poèmes de ce livre, dans leur travail de poda lírica, autrement dit d'épure et de stylisation, explorent, d'une façon ou d'une autre, les possibilités de l'épigramme. Car Lorca comprend parfaitement comment une forme traditionnelle, la copla, peut coïncider, pourvu qu'on la soumette à certaines distorsions, avec une sensibilité moderne fondée sur des formes poétiques brèves ${ }^{44}$.

Cette rencontre entre tradition et modernité, nous la voyons s'exprimer de façon exemplaire dans le poème «Puñal » (p. 156) avec sa double cuarteta subissant une distorsion due à la fois à la métrique (4/7/9/4 et 4/7/7/4) et à la présence d'une rime assonante oxytone et orpheline en /ó/ au deuxième vers de chaque quatrain (v. 2 corazón et v. 9 sol) tandis que le refrain nous offre un véritable calligramme où réapparaît la même rime assonante en /ó/. Car le refrain est un tercet qui dessine de façon stylisée les deux objets auxquels, dans le premier quatrain, renvoient le comparé el puñal et le comparant la reja del arado:

\section{El puñal \\ entra en el corazón \\ como la reja del arado \\ en el yermo.}

No.

No me lo claves

No.

En réalité, nous pourrions appliquer à de nombreux textes de Poema del Cante Jondo l'expression employée par Philippe Jaccottet pour désigner certains poèmes très brefs de Giuseppe Ungaretti, qu'il appelle des " poèmesinstants $"{ }^{45}$. Bien que chacune des quatre sections majeures semble narrer une histoire, chaque poème constitue au sein de sa section un instant déterminé appréhendé comme un tout. C'est pour cette raison que de nombreux poèmes commencent et s'achèvent avec les mêmes vers comme pour mieux suggérer leur aspect clos sur eux-mêmes. Mais comme l'écrit Jean-Marie Gleize à propos des formes poétiques brèves : "La clôture du texte, c'est l'ouverture infinie du sens [...]. Le texte court fonctionne comme un chiffre " ${ }^{46}$.

44. Ceci nous rappelle ce qu'écrit Alain Montandon dans son essai sur «les formes brèves » : "La brièveté est devenue, au XX $\mathrm{XX}^{\mathrm{e}}$ siècle, une des caractéristiques de la modernité, au point que l'on peut parler de brièveté comme forme caractéristique d'une certaine poésie après Baudelaire, Rimbaud et Mallarmé. », op. cit., p. 148.

45. La semaison (Carnets 1954-1967), Paris, N.R.F., Gallimard, 1971, p. 47.

46. Cité par A. Montandon, Op. cit., p. 147. 
C'est pourquoi il est très intéressant de remarquer que la section Gráfico de la petenera commence et s'achève avec des poèmes très semblables l'un à l'autre ("Campana» p. 174 et "Clamor" p. 183). Les deux poèmes s'ouvrent sur une sonnerie de cloches, voire un glas, et prennent fin avec le retour du silence. Cette relation spéculaire entre le premier poème et le dernier semble suggérer que toute la section pourrait être contenue ou se résumer dans "Clamor ", le dernier texte où la chanson chantée par la mort "sur sa vihuela " pourrait correspondre à toute cette série de poèmes lus ou entendus auparavant et qui forment l'ensemble de la section. Le poème bref fait ainsi de sa "clôture " une ouverture infinie car il fonctionne comme un " chiffre».

\section{POÉSIE ET THÉÂTRALITÉ : DU TABLADO AUX DIÁLOGOS}

Dans ce « retable » andalou qu'est Poema del Cante Jondo - on pourrait le dire, à plus forte raison encore, à propos de Romancero gitano -, les images et les rythmes supposent la représentation d'un espace-temps où vont se succéder différentes péripéties vécues par des êtres, évoqués souvent comme des personnages dont les voix surgissent parfois directement du texte, comme sans intermédiaire, face au lecteur comme si ce dernier était un simple spectateur. D'où une théâtralité constante mais qui se manifeste de façon différente entre Poema del Cante Jondo et Romancero gitano.

En réalité, la théâtralité semble étroitement liée à l'art poétique lorquien presque dès ses débuts. D’après Ian Gibson, Lorca, déjà en 1919, écrit plusieurs pièces courtes. En 1920, il représente pour la première fois sa première véritable pièce, El maleficio de la mariposa, et son échec ne l'empêche pas d'écrire en 1922, Retablillo de don Cristóbal o Titeres de cachiporra. Dès 1924, il travaille sur des pièces importantes Mariana Pineda et La zapatera prodigiosa et en 1926 sur Amor de Don Perlimplín con Belisa en su jardín.

De sorte que Lorca compose ces pièces en même temps que Romancero gitano, écrit entre 1923 et 1926 . Et pendant presque la même période, vers 1925, il compose une demi-douzaine de diálogos ou sketches graves, poétiques, parfois absurdes ou burlesques, tous insolites et très brefs, qui ne sont pas destinés à la scène, bien qu'ils appartiennent au genre théâtral ( $\mathrm{La}$ doncella, el marinero y el estudiante, El paseo de Buster Keaton, Quimera ...). Or, à cet ensemble de Diálogos, écrits quatre ans après les deux premiers volets de Poema del Cante Jondo, appartiennent " Escena del Teniente Coronel de la Guardia Civil " et "Diálogo del Amargo », ajoutés, en 1931, au recueil pour sa publication afin de le parachever et de lui donner plus de volume. 
Pendant les dix dernières années qu'il lui reste à vivre, Lorca, tout en composant des recueils importants, va consacrer chaque fois plus de temps au théâtre, à la fois comme directeur de la "Barraca " et comme auteur, puisqu'il écrit alors ses pièces les plus importantes (Bodas de sangre, Yerma, La casa de Bernarda Alba) et un "théâtre impossible ", avant-gardiste et surréaliste qui ne sera représenté que 40 ou 50 ans après sa mort (Asi que pasen cinco años et El público).

Tout nous conduit à penser que cette simultanéité entre les deux travaux créatifs de Lorca, la poésie et le théâtre, n'est pas qu'une simple coöncidence mais qu'ils se nourrissent tous deux l'un de l'autre et l'aident à définir sa quête ontologique et esthétique. Le propre poète nous le suggère quand il déclare, en 1934, dans un entretien : "El teatro fue siempre mi vocación. He dado muchas horas de mi vida al teatro. El teatro es la poesía que se levanta del libro y se hace humana. El teatro fue siempre mi vocación " pour ajouter ensuite : "El teatro necesita que los personajes que aparezcan en escena lleven un traje de poesía y al mismo tiempo que se les vean los huesos, la sangre " ${ }^{47}$.

Cet authentique acte de foi montre clairement que le théâtre lorquien se fonde sur la poésie et laisse supposer indirectement que celle-ci, à son tour, pour parvenir aux lecteurs transformés en public doit leur proposer des situations et des personnages horrorosamente trágicos ${ }^{48}$, ce qui n'empêche jamais, bien sûr, quelques touches d'humour. Si bien que nous pourrions inverser la phrase lorquienne et dire que para levantarse del libro y hacerse humana su poesia se hace teatro du moins dans Poema del Cante Jondo et Romancero Gitano.

Lorsque nous regardons la définition du mot "théâtre" dans le dictionnaire Le Robert, nous trouvons : "Art visant à représenter devant un public [...] une suite d'événements où sont engagés des êtres humains agissant et parlant ". Bien que Poema del Cante Jondo et Romancero gitano n’aient pas été écrits pour être mis en scène, cette définition du théâtre peut nous permettre de détacher quelques concepts ou éléments qui, si on les considère avec une relative souplesse, peuvent apparaittre dans ces recueils, nous invitant à y voir la présence d'une certaine "mise en scène " depuis le texte même, pour faire du lecteur le "spectateur mental » d'un certain type de «représentation " proche du théâtre quoique de façon mimétique. Les concepts ou éléments nécessaires qu'implique la définition du dictionnaire sont les notions de "représentation devant un public ", de "péripéties" et surtout de "personnages et de dialogues " puisqu'au théâtre l'action est presque essentiellement du récit de paroles au discours direct.

47. Conversaciones literarias, Op. cit., p. 673.

48. Ibid. 
Les deux « dialogues » ou courtes scènes qui parachèvent Poema del Cante Jondo appartiennent de plein droit au genre théâtral. C'est pour cette raison que dans l'édition française des œuvres complètes de Federico García Lorca pour la Pléiade, André Belamich les extrait du recueil pour les intégrer au $2^{\text {ème }}$ tome consacré aux œuvres dramatiques du poète. On peut aussi rappeler qu'il y a vingt ans, d'authentiques représentations théâtrales furent données de ces " dialogues ", qui ne furent pas de simples récitals. En effet, en 1986, le Centro dramático del Teatro María Guerrero les mit en scène sous la direction de Joan Baixas.

Néanmoins, dans Poema del Cante Jondo, la théâtralité ne se manifeste pas, à notre avis, que dans le dernier volet du triptyque, dont les caractéristiques théâtrales, comme nous le constaterons, sont plus qu'évidentes, mais également, d'une certaine manière, dans les quatre premières sections qui servent de fondations au livre, consacrée chacune à une forme poétique et musicale jonda.

Une des caractéristiques de Poema del Cante Jondo, comme nous avons déjà eu l'occasion de le constater, est l'absence d'un locuteur personnel omniprésent, de ce Moi sentimental et nostalgique, un peu romantique qui dominait Libro de poemas. Or, le propos des quatre sections majeures est « la mise en scène " des formes jondas, en créant pour chacune d'entre elles un espace qui corresponde à la section qui lui est attribuée avec une intrigue particulière, que nous avons déjà détaillée, où chaque poème devient une sorte de scène. Enfin, cet espace, ainsi créé mentalement à partir du texte, doit toujours conduire implacablement le lecteur-spectateur vers un dénouement funeste qui symbolise la quintessence de la Peine andalouse.

Les premiers poèmes de chaque section ("Paisaje ", «Evocación», "Arqueros" et "Campana ") fonctionnent un peu comme les didascalies d'un paratexte intermédiaire, chargées de suggérer un décor, à chaque fois différent mais toujours andalou (campo de olivos, tierra seca, monte pelado, calle, cueva, pueblo perdido en la Andalucía del llanto ou Sevilla lors de la Semaine Sainte).

Il est également intéressant d'observer dans ces poèmes l'emploi fréquent de vers entre parenthèses qui rappellent les didascalies d'un texte dramatique. C'est ainsi que nous trouvons dans "El grito" (p. 148) entre deux ayes une véritable didascalie porteuse d'un message visuel animé lié à un jeu de lumières: (Las gentes de las cuevas/ asoman sus velones) ou dans " Evocación " (p. 153) une autre didascalie entre parenthèses, chargée de compléter la toile de fond en donnant deux indications, l'une visuelle, l'autre auditive : Viento en el olivarl viento en la sierra). 
Une fois évoqué le décor, c'est-à-dire la toile de fond propre à chaque section, font irruption la musique et le cri, typiques du Cante Jondo, échos d'une histoire à chaque fois différente mais qui constitue toujours une allégorie de la Pena andaluza, destinés à préparer l'apparition d'une forme jonda particulière qui va entrer en scène puis disparaître. La trouvaille lorquienne réside probablement, comme nous l'avons déjà indiqué, dans la prosopopée des différentes formes (siguiriya, soleá, petenera) transformées ainsi en des personnages qui peuvent monter sur le tablado pour danser ou chanter et ensuite disparaître ou mourir en fonction des silences. La saeta est un cas à part car elle est déplacée ici sur le personnage de la saetera, la Lola, qui transforme l'oraison en un chant profane destiné, telles les flèches de Cupidon, à transpercer d'Amour ceux qui en sont la cible, qu'il s'agisse des toreritos, du barberillo ou d'autres saeteros.

Il convient, cependant, de s'interroger sur l'identité de la voix poématique, à qui ou à quoi peut-elle bien correspondre ici ? Suppose-t-elle toujours le même locuteur?

La plupart du temps c'est une voix anonyme et populaire, une voix qui semble liée à la tradition jonda, celle du propre texte devenu le public supposé de ce "retable ", le texte devenu lui-même le spectateur ou l'auditoire du spectacle qu'il produit. Cette voix peut, bien évidemment, être aussi celle du cantaor mais, à travers elle, vibre tout un chant andalou, par conséquent communautaire et identitaire qui suppose la présence active d'un public. Celui-ci crie, interpelle et, comme dans la tradition jonda, anime la siguiriya, la soleá ou la petenera qui défilent sur la scène ou sur le tablado, des voix qui peuvent être aussi celles d'une sorte de chœur :

\author{
¿Adónde vas, siguiriya, \\ con un ritmo sin cabeza? \\ ¿Qué luna recogerá \\ tu dolor de cal y adelfa? \\ («El paso de la siguiriya », p. 150)
}

ou

$$
\begin{aligned}
& \text { ¡Ay, petenera gitana! } \\
& \text { ¡Yayay petenera! } \\
& \qquad \text { («Falseta », p. 181) }
\end{aligned}
$$

Comme aucun véritable dialogue n'apparaît dans cette partie du livre - contrairement à ce qui se passe dans les deux courtes scènes du dernier volet, voire dans certains romances gitans -, le dramatique s'exprime lyriquement par l'emploi du discours direct, qui n'est manifeste que lorsque font irruption des exclamations, des interrogations ou des apostrophes adressées par une 
voix anonyme à un personnage explicite ou implicite. Ce qui est intéressant, c'est que cette voix anonyme, que nous identifions souvent à la voix de la tradition et qui peut inclure autant le cantaor que son public transformé en chœur, résonne parfois comme celle d'un personnage identifiable ou non du spectacle.

C'est ainsi que dans le poème «Puńal », p. 156, se fait entendre la voix d'un personnage inconnu :

No.

No me lo claves.

No.

future victime qui pressent une menace et dont le corps poignardé sera découvert par la suite dans le poème "Sorpresa ", p. 159, où l'on entendra la voix de celui qui le découvrira, communiquant à sa mère l'obsédante image de la scène :

Muerto se quedó en la calle
con un puñal en el pecho.
No lo conocía nadie
¡Cómo temblaba el farol!
Madre.
¡Cómo temblaba el farolito
de la calle!

Mais cette vision, contrairement à ce que laisse entendre le titre du poème, n'est pas une «surprise " pour tout le monde puisqu'elle avait été annoncée, deux poèmes auparavant, dans «Encrucijada », p. 157. Une vision qui alors se manifestait déjà avec les détails du futur crime : l'évocation anticipée du puñal, de la calle, d'un farol et de son temblor. Et tout nous conduit à penser, si nous suivons la logique de cette représentation particulière, que ce locuteur personnel et "voyant " était déjà la soleá avec son regard doué d'ubiquité : Por todas partes/ yo/ veo el puñall en el corazón.

Nombreuses sont les voix de personnages inconnus qui font irruption au milieu d'un texte. C'est le cas de la mère qui dans le poème "El silencio ", p. 149, après "El grito ", réclame l'attention de son fils : Oye, hijo mío, el silencio./ Es un silencio ondulado. Un fils qui, ici, peut être le public lui-même qui se doit d'être attentif à l'arrivée de la «siguiriya » sur le point d'entrer en scène.

En revanche, dans les poèmes « Paso », p. 170 et « Saeta », p. 171, la voix qui s'exprime est sans nul doute celle du saetero qui incite à adorer l'image de la Vierge ou le paso portant la statue du Cristo moreno, en annonçant avec emphase sa venue : jMiradlo por dónde viene!, en ayant recours à une formule 
qui rappelle celle qu'employaient les juglares dans les romances viejos : $;$ Helos, helos por do vienen/ con toda la su compaña...! (in Los Infantes de Lara).

Mais, nous découvrons ensuite que ce saetero pourrait être en fait, une saetera comme la Lola qui apparaît dans " Balcón », p. 172, le poème suivant : La Lolal canta saetas. Des saetas qui vont aveugler les saeteros soudain épris de cette Lola sensuelle : Pero como el amor/ los saeteros están ciegos. Une Lola dont la sensualité nous sera confirmée dans le poème "Lola" de la section Dos muchachas. Mais pour l'instant, nous pouvons remarquer grâce à l'irruption inopinée de ce personnage féminin un habile et irrespectueux détournement de l'amour divin vers l'amour profane. Encore que cette laïcisation du thème de la saeta ait pu être amenée antérieurement grâce à une certaine distanciation humoristique à l'égard de l'orthodoxie du cérémonial religieux. De sorte qu'il convient de rappeler que, quatre poèmes plus tôt, une voix inconnue mêlait le profane au religieux pour suggérer alors une vision quelque peu fantaisiste et même burlesque de la "procession", p. 169 : Por la calleja vienen/ extraños unicornios./../ Fantásticos Merlines/y el Ecce Homo,/ Durandarte encantado, / Orlando furioso.

Dans Gráfico de la petenera, dès "Camino ", le second poème (p. 176) de la section, une voix anonyme semblable à celle d'un chœur ou d'un coryphée, comme dans les tragédies grecques, commente l'action pour obliger le lecteur-auditeur à s'interroger sur le destin des cien jinetes enlutados:

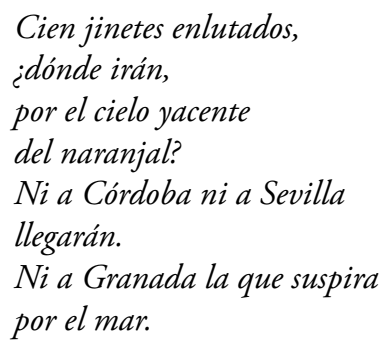

Un destin funeste qui d'emblée semble prédéterminé puisque leur voyage ne doit les conduire vers aucune ville connue mais vers un laberinto de cruces, lieu où sera enterrée la Petenera dans le poème "Falseta ", p. 181, et où eux-mêmes, amoureux infortunés, connaîtront, à cause de cette femme maléfique, désignée comme la perdición de los hombres, un rêve éternel comme le suggère le poème "De profundis ", p. 182, dont le titre renvoie à une prière pour les morts :

Los cien enamorados duermen para siempre

bajo la tierra seca. 
L'évocation d'un décor, orienté par des didascalies qui apportent des messages visuels animés et auditifs, la personnification des formes poétiques et musicales ainsi que les interventions répétées d'un discours direct, susceptible de diverses interprétations qui vont du simple personnage aux commentaires d'un chœur en passant par une voix anonyme, celle du propre texte transformé en public, sans oublier les péripéties successives qui forment le nœud et le dénouement de chaque section, tout cela contribue à donner à ce recueil une certaine théâtralité. Une théâtralité qui se prolonge dans certains poèmes d'autres sections comme dans "Amparo", p. 185, qui s'ouvre et se referme sur une apostrophe adressée à la chaste et recluse Amparo par le personnage d'un amoureux malheureux et anonyme :

\section{Amparo, \\ iqué sola estás en tu casa vestida de blanco!}

ou encore dans le poème "Baile ", p. 198, où surgit, parmi les cuartetas qui évoquent la danse obscène de la vieille Carmen, la voix scandalisée d'autres personnages, les mères bien pensantes qui veillent sur l'honneur de leurs filles : iNiñas/ corred las cortinas!.

Dans les deux diálogos qui composent le dernier volet de Poema del Cante Jondo, «Escena del Teniente Coronel de la Guardia Civil " et "Diálogo del amargo ", la théâtralité s'impose, à présent, d'emblée comme forme primordiale, autrement dit comme le genre propre à ces textes qui peuvent se définir comme un " théâtre bref et poétique ". Le genre théâtral apparaît immédiatement par la simple disposition typographique de ces tableaux qui s'organisent autour d'un texte dialogué accompagné d'importantes didascalies où sont présents les trois types de paratexte inhérents au genre théâtral :

- le paratexte liminaire concernant le titre

- le paratexte intermédiaire évoquant l'espace scénique, Cuarto de banderas pour le premier texte et Campo pour le second.

- le paratexte interstitiel ${ }^{49}$ qui inclut les noms des personnages avant chaque réplique ainsi que les indications scéniques - nombreuses ici-, écrites entre parenthèses et en italiques et qui se réferent aux jeux de lumière, aux déplacements, à la gestuelle ou aux mimiques des personnages, aux

49. Nous reprenons ici la terminologie et la typologie employées par Jean-Marie Thomasseau à propos du texte " disdascalique ", ce " texte à voir " purement informatif qui doit permettre la mise en scène, dans "Les différents états du texte théâtral ", in Pratiques, $\mathrm{n}^{\circ}$ 41, mars 1984, p. 99-121. 
tons de leur voix et aux sons en général, sans compter l'indication de divers accessoires ou effets scéniques.

Mais ce qui est intéressant, c'est qu'à présent c'est le poétique qui devient la caractéristique définitoire de ce théâtre bref qui, dans les deux cas, se fonde sur un seul tableau avec trois ou quatre personnages et une action relativement simple, tragique et onirique. Un théâtre poétique bref qui annonce déjà certains thèmes ou poèmes du Romancero Gitano ; il suffit de penser au Romance de la Guardia Civil et à celui del Emplazado où apparaît également le personnage d'Amargo. De sorte qu'à présent, nous sommes dans une situation inverse à celle vue jusqu'ici, puisqu'au lieu de chercher le théâtral dans le poétique, nous allons essayer de mettre en évidence le poétique au sein de ce qui se présente d'emblée comme théâtral.

Nous pouvons observer que dans les deux "dialogues " les personnages sont des archétypes. C'est ainsi que dans le premier tableau, nous assistons au futur affrontement mythologique du Romancero Gitano entre l'ordre cruel, deshumanisé, insensible, présomptueux et ridicule par manque d'humour de la Garde Civile et la fantaisie créative et récréative du gitan, incarnation idéale à la fois du marginal et de l'artiste, tous deux persécutés, martyrisés et sacrifiés par la société incarnée par ses acolytes et ses sbires. Quant au deuxième tableau, il nous introduit progressivement, à partir d'une conversation énigmatique et inquiétante, dans la conscience aiguë et obsédante de la mort et de la contingence inhérente à la nature humaine.

Mais, d'où provient exactement le poétique dans ces deux textes dramatiques? Il ne procède pas seulement des canciones qui servent de prolongement à chacun des deux tableaux ni des cuartetas qui, comme un bref romance, apparaissent dans le premier tableau - possible trace d'un romance gitan non achevé selon une lettre adressée à Jorge Guillén -, ni même de la copla qui ouvre avec une voix anonyme le second tableau où les pies quebrados laissent présager, avec la présence des adelfas, le destin funeste d'Amargo.

Le poétique se manifeste avant tout dans des situations oniriques qui ne donnent pas au lecteur-spectateur un nœud et un dénouement fermés sur eux-mêmes mais bel et bien ouverts, hautement symboliques à partir d'images plastiques, qui sont celles que suscitent les personnages et les effets visuels et auditifs présents sur la scène. Mais il est intéressant de constater que là où s'insère le plus le "langage poétique " - c'est-à-dire les images et les métaphores - c'est précisément au sein des didascalies, normalement destinées à la mise en scène et à l'origine de signes non verbaux lors d'une éventuelle représentation. C'est ainsi que dans le premier tableau le paratexte interstitiel signale la présence d'un fusil de sombra submarina ou de el alma de 
tabaco y café con leche del Teniente Coronel (que) sale por la ventana tandis que dans le deuxième "tableau ", il nous indique que le cri du chant d'Amargo pone un acento circunflejo sobre el corazón de los que lo han oído, suggérant ainsi, avec le signe angulaire de l'accent, l'aspect pénétrant et effrayant du cri. De sorte que dans ces didascalies particulières, le langage ne fonctionne pas seulement d'une façon référentielle mais avant tout métaphorique.

Par ailleurs ces didascalies peuvent parfois suggérer un gros plan presque cinématographique sur un accessoire comme sur l'étiquette d'une boîte de cigares: (Romeo y Julieta, celeste, blanco y oro, se abrazan sobre el jardin de tabaco de la caja de puros...), p. 206, ou sur le regard des personnages, caractérisé métaphoriquement ou par un mot familier : (La mirada de mulo joven del gitanillo ensombrece y agiganta los ojirris del Teniente Coronel de la Guardia Civil). Cela montre que Lorca joue constamment sur le langage employé dans les indications scéniques, jusqu'à créer, à partir du qualificatif salomónico de columna salomónica, le néologisme salomoniza pour évoquer l'ombre torse du cheval : (El camino ondulante salomoniza la sombra del animal.)

Enfin, ces didascalies poétiques non seulement permettent, comme au sein de n'importe quelle pièce, d'apporter des messages visuels animés : (Llegan tres jovenes con anchos sombreros), p. 211, et d'autres auditifs: (Ranas y grillos hacen la glorieta del estío andaluz)", p. 212, mais elles sont aussi capables de nous faire sentir le contraste entre deux sons, l'un proche, intime, devenu soudain inquiétant et annonciateur du second : (...Su gran reloj de plata le suena oscuramente en el bolsillo a cada paso) et l'autre, lui, extérieur et immédiat : (Un jinete viene galopando por la carretera.), p. 213. Didascalie synesthésique d'une grande précision qui permet d'associer sur le champ le cavalier de la mort au temps qui s'écoule inexorablement.

Quant aux répliques, nous pouvons remarquer qu'elles privilégient toujours l'insolite, qu'il soit burlesque, inquiétant, poétique ou tragique. Dans le deuxième tableau, le poétique, au sein des répliques, se manifeste surtout à travers le motif du "couteau " - écho du puñal de Poema de la soleá - et qui, comme dans les romances gitans fonctionne comme une image métaphorique clef, sorte de matrice poétique qui va se développer et proliférer à travers tout le dialogue entre le cavalier et Amargo, avec un aspect à la fois effrayant et fascinant qui associe beauté et horreur.

Dans «Escena del Teniente Coronel de la Guardia Civil », la poésie s'incarne principalement dans le personnage du gitan et dans son langage, lequel va retentir comme un coup de feu mortel pour le lieutenantcolonel qui, lui, tire son identité d'un langage prosaïque, présomptueux, monothématique, fortement hiérarchisé et essentiellement conatif. La poésie, 
langage subversif par excellence, apparaît clairement comme possédant un pouvoir de destruction sur l'autre monde, le monde matérialiste, bien que le prix à payer en soit, comme le suggère le destin malheureux du gitan rossé à mort, le sacrifice de celui qui l'emploie et le revendique.

Ce pouvoir libérateur de la poésie se fonde, bien évidemment, sur les images employées par le gitan, mais ce qui me paraît particulièrement intéressant et que personne à ma connaissance n'a signalé, c'est que ces images semblent suivre une discrète bien qu'authentique et subtile métrique. Malgré l'aspect typographiquement non versifié de ce texte en prose, nous pouvons observer que presque toutes les réponses du gitan sont, lorsqu'elles sont supports d'images, d'authentiques octosyllabes, ces vers populaires qui servent de fondement au Romancero gitano (p. 208) :

En/ ell puen/tel del los/ ríl os
7
U/nal to/rrel del calnella
7
A/zulfrey/ ro/saen/ mis/ $\underline{\text { lalbios }}$
7
En/ elnelro/ ten/goalzahar
7
Yl nalran/jas/ en/ lal nielve
7

Une autre réplique du gitan qui précède un octosyllabe constitue, cette fois, un véritable alejandrino $(7+7)$ :

\section{Hein/ven/taldou/nas/ allas// pa/ral vollar, / y/ vue/lo \\ 6 \\ 6}

Or, il est particulièrement significatif que ces "deux mètres" qui définissent l'invention poétique du gitan, soient complétés (p. 209), juste après le premier ; ay! douloureux du lieutenant-colonel, par deux nouvelles phrases d'affilée qui supposent une émancipation par rapport à ce qui a été dit avant, et qui, cependant, était déjà un symbole de liberté. Car, à présent, ce qui ne manque pas d'intérêt, si nous considérons également d'un point de vue métrique ces deux nouvelles phrases, c'est qu'elles constituent, toutes deux, bel et bien une émancipation par rapport au patron métrique précédent : en effet, les deux nouveaux « mètres " qu'elles nous proposent - un pentadécasyllabe $(8+7)$ et un ennéasyllabe - n'ont chacun qu'une seule syllabe surnuméraire si l'on considère les deux types métriques offerts auparavant : 
POEMA DEL CANTE JONDO: RÉÉVALUATION D’UNE POÉTIQUE EN DEVENIR

Aun/quel nol nelcelsiltoallas,// por/quel vuello/ sin/ elllas.
7

Nu/bes/ $y /$ alni/llos/ en/ mi/ san/gre

8

Nous trouvons ici une parfaite adéquation entre la forme et le contenu puisque " métriquement » nous avons une émancipation qui n'a cette valeur que parce qu'elle ne s'écarte que très légèrement des «mètres " précédents au point de signifier à la fois davantage de liberté et souvenir affranchi du modèle antérieur.

Pour Lorca poésie et théâtralité sont toujours indissociables même dans des aspects qui peuvent passer inaperçus comme cette "métrique masquée ", insérée au sein de ce texte dramatique en prose. Or, s'il est bien un genre qui bientôt allait pouvoir permettre à Lorca de combiner de façon idéale et exemplaire poésie et théâtralité, c'est très certainement le romance, d'où les liens très étroits qui semblent unir Poema del Cante Jondo et Romancero Gitano.

Deuxième recueil écrit par Lorca, Poema del Cante Jondo s'affirme, pour nous, comme le livre d'une poétique en devenir, autrement dit d'une poétique qui inscrit en elle-même ses propres potentialités, lesquelles doivent lui permettre d'évoluer et de se prolonger à travers d'autres recueils à venir. Après Libro de poemas qui fixe d'emblée un univers lorquien dont les symboles et les mythes sont déjà en germe et ne demandent qu'à se développer, Poema del Cante Jondo apporte une véritable réflexion sur l'écriture poétique, faite de lucidité et d'exigence. Ce recueil s'inspirant avec intelligence de la tradition populaire parvient à éviter les pièges d'un Moi par trop effusif et sentimental en prônant une poétique de l'épure, de «l'élagage lyrique ». C'est ainsi que la voix poématique se met elle-même en scène afin de nous montrer comment "le chant - comme le dit Marie-Claire Zimmermann - secrète les lois de l'écriture poétique " ${ }^{50}$.

Mais ces «lois" ne sont possibles que parce que la tradition populaire n'est jamais ici "folklorisme ", c'est-à-dire simple expression pittoresque de topiques éculés, mais bien au contraire une constante possibilité de se renouveler grâce à une lecture inédite d'un héritage passé néanmoins ouvert à la modernité. Poema del Cante Jondo est sans nul doute le parfait champ d'application de cette relecture de Góngora, devenue une théorie lorquienne

50. Poésie espagnole moderne et contemporaine, Paris, Dunod, 1995, p. 72. 
de la métaphore et que le poète parvient - cinq ans plus tard et non sans quelques efforts préalables - à exprimer, dans un langage spéculatif et jamais doctrinaire, à travers La imagen poética de don Luis de Góngora. Or, cette image poétique, parfois formellement si proche des préoccupations d'autres poètes, se déclarant, eux, ouvertement avant-gardistes, Lorca ne peut l'atteindre ici qu'au prix d'une véritable ascèse langagière fondée sur une autre relecture inédite, celle de la copla, cette forme brève fondée sur la concentration et la concision.

Une relecture qui lui permet d'inventer une métrique plus libre, sans jamais céder pour autant à un vers-librisme facile, car dans Poema del Cante Jondo, le vers doit se prêter, avant tout, aux inflexions d'une "prose chantée " provenant d'une prosodie subtile et jamais systématique, qui semble, comme nous avons essayé de le montrer, née d'une adaptation "métricienne " de l'enharmonismo. Lorca a toujours une approche musicale du vers qui ne peut ignorer la mesure, laquelle semble même, comme nous l'avons vu, émerger discrètement et subtilement à la fin d'un texte en prose comme "Escena del Teniente Coronel de la Guardia Civil », à travers les répliques du gitano.

Nous aurions pu, bien évidemment, insister davantage sur le gitanismo et l'Andalousie - ces mythes essentiellement lorquiens - qui sont les aspects que l'on serait tenté de relier d'emblée au Poema del Cante Jondo. Des aspects qui furent, cependant, trop souvent l'objet de malentendus entre les textes lorquiens et leurs lecteurs, qu'il s'agisse pour ces derniers de les apprécier avec un engouement immodéré ou de les rejeter violemment. N'oublions pas que c'est presque sous la pression de Rafael Martínez Nadal que Lorca accepte, en 1931, de publier finalement Poema del Cante Jondo. Car, après le succès populaire exceptionnel de Romancero gitano, Lorca subit aussi, à son sujet, de violentes et douloureuses critiques de la part de certains de ses amis les plus proches qui lui font craindre d'être enfermé, à ses dépens, dans « un mythe de la gitanerie ", totalement étranger à ce à quoi aspire profondément sa poésie. C’est le cas de Buñuel qui rejette en bloc et sans ménagement le livre ou, encore, de Dalí qui, bien qu'il lui reconnaisse certaines qualités, écrit dans une lettre adressée à Sebastián Gasch, en novembre 1928, que lo importante es lo latente y no lo realizado et qui n'hésite pas, lui aussi, à déclarer : Del Romancero me repugna todo lo que tiene de trágico, de gitano ${ }^{51}$. Des propos qui ne font que confirmer une autre lettre, envoyée dès la publication du recueil par le peintre catalan, cette fois à Lorca lui-même, où

51. In Federico García Lorca. 1. De Fuente Vaqueros a Nueva York, 1898-1929, de Ian Gibson, Barcelona, Grijalbo, 1985, p. 571. 
il lui écrit que son livre se mueve dentro de la ilustracion de los lugares comunes mas estereotipados $i$ mas conformistas ${ }^{52}$.

La suite nous la connaissons, une profonde déception, voire dépression, un séjour de presque un an aux USA dont il rapporte une œuvre majeure aux accents "surréels ", Poeta en Nueva York, avant de renouer avec des recueils où se mêlent à nouveau tradition et modernité, qu'il s'agisse de l'élégie avec Llanto por Ignacio Sánchez Mejias, des Gacelas et Casidas de Diván del Tamarit, voire des Sonetos del amor oscuro. Des livres où la tradition est constamment revisitée pour répondre aux questionnements ontologiques et esthétiques du poète. C'est pourquoi nous pensons que si l'incompréhension injuste - faite d'envie et d'une certaine ignorance ou d'un simple refus de questionner réellement l'écriture poétique dont ont fait preuve certains de ses proches vis-à-vis de Romancero gitano - a pu faire craindre à Lorca de publier Poema del Cante Jondo, celle-ci ne doit pas oblitérer ce que ce livre a apporté non seulement au Romancero gitano mais à l'écriture lorquienne en général, et ce jusque dans des œuvres apparemment éloignées de ces thèmes.

Même la Pena andaluza - personnage principal de Poema del Cante Jondo - que Lorca définit dans sa conférence-récital sur Romancero gitano comme una lucha de la inteligencia amorosa con el misterio que la rodea y no puede comprender ${ }^{53}$ est un questionnement ontologique et esthétique qui demeure pertinent quelles que soient les orientations de son écriture poétique.

Enfin, n'oublions pas non plus que Poema del Cante Jondo, à travers ses quatre sections majeures et surtout grâce à l'ajout postérieur des deux " dialogues " du dernier volet, confirme les liens entre poésie et théâtralité qui, dès lors, vont se lier inextricablement au point d'être à l'origine d'une des œuvres dramatiques les plus importantes du $\mathrm{xx}^{\mathrm{e}}$ siècle. Poema del Cante Jondo est donc bien pour nous un recueil essentiel au sein de l'œuvre poétique lorquienne et dont nous n'avons que commencé à réévaluer - c'est du moins ce que nous espérons - l'importance de la poétique.

52. Ibid., p. 567. Nous reproduisons l'orthographe fautive de Dalí en castillan.

53. Op. cit., p. 340. 